\title{
JG|U
}

Gutenberg School of Management and Economics \& Research Unit "Interdisciplinary Public Policy" Discussion Paper Series

\section{The Invariant Distribution of Wealth and Employment Status in a Small Open Economy with Precautionary Savings}

Christian Bayer, Alan D. Rendall and Klaus Wälde

November 28, 2018

Discussion paper number 1822

Johannes Gutenberg University Mainz

Gutenberg School of Management and Economics

Jakob-Welder-Weg 9

55128 Mainz

Germany

https://wiwi.uni-mainz.de/ 
Contact details

Christian Bayer

Weierstrass Institute

Mohrenstr. 39

10117 Berlin

Germany

christian.bayer@wiasberlin.de

Alan D. Rendall

Institute of Mathematics

Johannes Gutenberg University Mainz

Staudingerweg 9

55128 Mainz

Germany

rendall@uni-mainz.de

Klaus Wälde

Gutenberg University Mainz

Johannes Gutenberg School of Management and Economics

Jakob-Welder-Weg 4

55128 Mainz

Germany

waelde@unimainz.de

www.waelde.com

All discussion papers can be downloaded from http://wiwi.uni-mainz.de/DP 


\title{
The Invariant Distribution of Wealth and Employment Status in a Small Open Economy with Precautionary Savings
}

\author{
Christian Bayer $^{(a)}$, Alan D. Rendall ${ }^{(c)}$ and Klaus Wälde ${ }^{(c)}$ \\ ${ }^{(a)}$ Weierstraß Institute Berlin \\ ${ }^{(b)}$ Institute of Mathematics, Johannes Gutenberg University Mainz \\ ${ }^{(c)}$ Department of Economics, Johannes Gutenberg University Mainz, CESifo and IZA ${ }^{1}$
}

November 28, 2018

\begin{abstract}
We study optimal savings in continuous time with exogenous transitions between employment and unemployment as the only source of uncertainty in a small open economy. We prove the existence of an optimal consumption path. We exploit that the dynamics of consumption and wealth between jumps can be expressed as a Fuchsian system. We derive conditions under which an invariant joint distribution for the state variables, i.e., wealth and labour market status, exists and is unique. We also provide conditions under which the distribution of these variables converges to the invariant distribution. Our analysis relies on the notion of T-processes and applies results on the stability of Markovian processes from Meyn and Tweedie (1993a, b,c).
\end{abstract}

JEL Codes: C62, D91, J63

Keywords: uncertainty in continuous time, counting process, existence, uniqueness, stability

\section{Introduction}

Dynamic and stochastic models are widely used for macro economic analysis and also for many analyses in labour economics. When the development of these models started with the formulation of stochastic growth models, a lot of emphasis was put on understanding formal properties of these models. Does a unique solution exist, both for the control variables and general equilibrium itself? Is there a stationary long-run distribution (of state variables being driven by optimally chosen control variables) to which initial distributions of states converge? The literature employing continuous-time models only initially put some emphasis on looking at stability issues (Merton, 1975; Bismut, 1975; Magill, 1977; Brock and Magill, 1979; Chang and Malliaris, 1987).

In very recent years, continuous-time idiosyncratic risk models allowing to study wealth distributions and, partially, their evolution over time have become very popular (see e.g. Benhabib, Bisin and Zhu, 2016, Nirei and Aoki, 2016, Achdou et al., 2017, Aoki and Nirei, 2017, Cao and Luo, 2017, Kaplan et al., 2018, Khieu and Wälde, 2018, Nuño and Moll, 2018). This does not mean, however, that all formal problems have been solved. In fact, an existence proof for policy functions and for a unique invariant wealth distribution is missing, as well as a proof for the stability of this invariant wealth distribution. (See section 2 for a more detailed discussion.)

\footnotetext{
${ }^{1}$ Christian Bayer: Weierstrass Institute, Mohrenstr. 39, 10117 Berlin, Germany. christian.bayer@wiasberlin.de. Alan D. Rendall: Institute of Mathematics, Johannes Gutenberg University Mainz, Staudingerweg 9, 55128 Mainz, Germany. rendall@uni-mainz.de. Klaus Wälde: Gutenberg University Mainz, Johannes Gutenberg School of Management and Economics, Jakob-Welder-Weg 4, 55128 Mainz, Germany. waelde@unimainz.de, www.waelde.com. We are grateful to William Brock, Martin Hanke-Bourgeois, Benjamin Moll, Manuel Santos, John Stachurski and Stephen Turnovsky for comments and suggestions. Klaus Wälde acknowledges generous financial support from the Gutenberg Research Council.
} 
The goal of this paper is twofold: First, we introduce mathematical tools for analysing existence of the consumption paths in models of this type. Individuals have constant relative risk aversion and an infinite planning horizon. Their wealth follows a stochastic path, as does their labour market status. Individuals can smooth consumption by accumulating wealth.

Second, we introduce methods from the stochastics literature to study the existence, uniqueness and stability of distributions described by controlled stochastic processes. We use these methods to analyse stability properties of a precautionary-savings model. We analyse under which conditions an invariant (stationary) distribution for wealth and employment status exists, is unique and when the distribution is stable in the sense that an initial distribution converges to the unique invariant one. The corresponding theorem is proven.

The framework for our analysis consists of a one-person optimal consumption-saving problem. Properties of implied consumption, wealth and employment dynamics can (almost trivially) be aggregated to represent aggregate consumption, wealth and employment dynamics of a small open economy. The individual can accumulate a riskless asset. The employment status (and thereby labour income) of the individual switches randomly (according to exogenous arrival rates) between two levels. Factor rewards (labour income levels and the interest rate) are (endogenously) fixed in our model. The underlying two-state Markov process implies that state-variables are piecewise deterministic. Wealth and consumption evolve smoothly in between changes of the employment status.

There are two sets of findings. Our existence proof for the policy function exploits the fact that optimal behaviour in a piece-wise deterministic system ${ }^{2}$ is described by an ordinary differential equation system. This system is particularly interesting as it has features many models of precautionary saving in continuous time share. First, there is a singularity both at the lower bound of the relevant range of the state variable (the 'natural borrowing limit') and at the upper bound (the temporary steady state). Second, while one boundary condition is almost exogenous (the 'natural borrowing limit'), the second boundary condition (the temporary steady state) is endogenous. We actually find it most convenient for our proof to proceed in two steps. We first prove existence for a regularized system (i.e. we consider a reduced set where singularities are absent), which is interesting in its own. We then enlarge the regularized system. While this is simple for the lower bound, we refer to an argument based on Fuchsian systems (Kichenassamy, 2007, Rendall and Schmidt, 1991) to enlarge the regularized system also with respect to the upper bound.

We find it most convenient to build the proof of the existence and uniqueness of an invariant distribution and its ergodicity, i.e. of convergence to the said distribution, on the work of Meyn and Tweedie (1993 a,b,c) and Down et al. (1995). ${ }^{3}$ Their work is especially useful for understanding properties of systems driven by jump processes. ${ }^{4}$ One crucial component of our proofs concerning the distribution of wealth is a smoothing condition. As we allow for counting processes, we have to use more advanced methods based on $T$-processes than in the case of a stochastic differential equation driven by a Brownian motion. In the latter case the strong smoothing properties of Brownian motion can be used to obtain the strong Feller property. In

\footnotetext{
${ }^{2}$ This is a property of a large class of economic models with random transitions between employment states (search and matching models) or for many innovation and growth or business cycle models where innovations occur at exponentially distributed random points in time often determined by Poisson processes.

${ }^{3}$ The theory we will employ below provides a useful contribution to the economic literature as the latter, as presented below, focuses on related, but different methods. For one, we treat Markov processes in continuous time, while references in the macro-economic literature in the context of Markov-process stability are mostly related to discrete time. But even in discrete time, the theory of $T$-processes of Meyn and Tweedie (a weaker version of strong Feller processes), seems new in the economics literature. While relying on other results from Meyn and Tweedie (1993a), Kamihigashi and Stachurski (2012, 2013), for instance, infer stability from order mixing properties instead.

${ }^{4}$ These methods are also used for understanding how to estimate models that contain jumps (e.g. Bandi and Nguyen, 2003) or for understanding long-term risk-return trade-offs (Hansen and Scheinkman, 2009).
} 
this sense, the corresponding analysis will often be more straightforward than the one presented here. For the wealth-employment process of our model, we find that the wealth process is not smoothing and the strong Feller property does not hold. However, for the economically relevant parameter case (the low-interest rate regime), we can still show a strong version of recurrence (namely Harris recurrence) by using a weaker smoothing property, and thus obtain uniqueness of the invariant distribution. Ergodicity is then implied by properties of discrete skeleton chains.

The structure of our paper is as follows. The next section relates our analysis to the literature. Section 3 presents the consumption-saving problem, derives the differential equations describing optimal consumption between jumps and aggregates consumers of a small open economy. Section 4 proves existence and properties of an optimal consumption function. Section 5 proves existence and uniqueness of an invariant measure for the state variables together with convergence to the long-run invariant distribution. Before this is done, section 5 also provides some general background to stochastic processes in continuous time which are needed for our main proofs. The final section concludes.

\section{Related literature}

We first relate our analysis to the general economic background, i.e. the discrete-time BewleyHuggett-Aiyagari model. We then turn to the continuous-time stochastic growth literature more generally. Finally, we relate our work to the very recent continuous-time literature that allows for precautionary saving. While the latter is mostly in general equilibrium, the maximization problems employed in these models are very similar if not, in some cases, identical to our structure. We therefore see our maximization problem as a generic example of many of the maximization problems in these models. As a consequence, our methods would be good starting points to understand existence and stability issues in general equilibrium as well.

Our analysis was originally motivated by precautionary saving models of the Bewley-HuggettAiyagari type (see Bewley, 1986, Huggett, 1993, and Aiyagari, 1994). Huggett (1993) analyses an exchange economy with idiosyncratic risk and incomplete markets. Individual endowment in each period is either high or low, following a stationary Markov process. Agents can smooth consumption by buying and selling an asset. Huggett provides existence and uniqueness results for the value function and the optimal consumption function and shows that there is a unique long-run distribution function to which initial distributions converge. Regarding stability, he relies on the results of Hopenhayn and Prescott (1992). An overview of the various directions the precautionary savings model took is provided by Heathcote et al. (2009). Our household maximization problem can be seen as the continuous-time version of the saving problem in Huggett (1993). As the recent continuous-time literature on precautionary models has shown (see below for more background), working in continuous time yields many novel results and insights.

Concerning the continuous-time stochastic growth literature, the starting point is Merton's (1975) analysis of the continuous-time stochastic growth model. For the case of a constant saving rate and a Cobb-Douglas production function, the "steady-state distributions for all economic variables can be solved for in closed form". No such closed form results are available of course for the general case of optimal consumption. Chang and Malliaris (1987) also allow for uncertainty that results from stochastic population growth as in Merton (1975) and they assume the same exogenous saving function where savings are a function of the capital stock. They follow a different route, however, by studying the class of strictly concave production functions (thus including CES production function and not restricting their attention to the Cobb-Douglas case). They prove "existence and uniqueness of the solution to the stochastic Solow equation". They build their proof on the so-called reflection principle. More work on growth was undertaken by Brock and Magill (1979) building on Bismut (1975). Magill (1977) 
undertakes a local stability analysis for a many-sector stochastic growth model with Brownian motions using methods going back to Rishel (1970). All of these models use Brownian motion as their source of uncertainty and do not allow for exponentially distributed jumps or counting processes.

There is a recent continuous-time macro-labour literature where precautionary saving behaviour plays a central role for understanding the distribution of wealth. Lise (2013) studies a partial equilibrium search model in the Burdett-Mortensen tradition allowing for on-the-job search. Achdou et al. (2014) survey (general equilibrium) continuous-time models in macroeconomics with a focus on partial differential equations emphasizing theoretical open ends like the lack of proofs of existence and uniqueness. They also present a precautionary saving model where uncertainty results from Brownian motion. Benhabib, Bisin and Zhu (2016) study the wealth distribution in a model with stochastic death. The quantitative fit for the upper-tail of the wealth distribution was studied by Nirei and Aoki (2016), Aoki and Nirei (2017) and Cao and Luo (2017). Kaplan et al. (2018) target moments of the wealth distribution and match top shares in a New Keynesian model where households die according to an Poisson arrival rate. Ahn et al. (2018) describe numerical methods for continuous-time models that would allow to solve idiosyncratic risk models that include aggregate shocks. Nuño and Moll (2018) follow a central planner approach to understanding wealth distributions. Achdou et al. (2017) discuss mass points in a continuous time Bewley-Huggett-Aiyagari model. Khieu and Wälde (2018) quantitatively study the dynamics of the wealth distribution to understand the NLSY 79 cohort. $^{5}$

What is known about the existence of policy functions or wealth distributions in these analyses? Achdou et al. (2014) report the absence of central existence proofs and refer to (what is now) Achdou et al. (2017). The latter sketch analytical proofs for existence and uniqueness of the stationary distribution. We prove the existence of a policy function. We also provide an alternative and fully probabilistic proof of existence and uniqueness of stationary distributions. In addition to the distinction between analytic vs. probabilistic approaches, we establish ergodicity of the system, i.e., convergence in distribution for arbitrary initial distributions to the unique stationary distribution. In that sense, the system under consideration is stable with respect to the wealth and employment distribution of one individual and also for a whole population whose labour income and interest rate are constant. ${ }^{6}$ As is well-known from deterministic systems (think of stable cycles), the existence and uniqueness of a steady state does not imply that this is the relevant long-run state of a system. Given our stability proof, we now know that the long-run unique stationary distribution is the one to which the system converges. Interestingly, Achdou et al. (2017) find that the continuous time Bewley-HuggettAiyagari model displays a mass-point at the lower end. When we prove existence, uniqueness and stability of the stationary distribution, we do not require the existence of a density. Our stability proof is valid when there is a mass point and when there is no mass point (see Theorem 5.18 and especially Lemma 5.20).

\footnotetext{
${ }^{5}$ These are by far not the only continous-time models with precautionary saving. Scheinkman and Weiss (1986) study a precautionary savings setup with a borrowing constraint when the interest rate is zero (e.g. for holding cash) in a two-type economy. Lippi et al. (2015) extend their framework to time-varying money supply. There are also many papers that employ an instantaneous utility function of a CARA structure (as opposed to our CRRA structure), see e.g. Wang (2007) and references therein. Wang et al. (2013) employ non-expected recursive utility to study the effects of risk-aversion and intertemporal substitution on optimal consumption behaviour. The effect of altruism in a setup with overlapping generations is studied by Barczyk and Kredler (2014). Continuous time models are also heavily employed in the finance literature. As an example, Raimondo (2005) proves existence of equilibrium in a model with incomplete and with complete markets. Anderson and Raimondo (2008) prove dynamic completeness of the equilibrium price process.

${ }^{6}$ As written above, we provide proofs for the saving problem of the household which are also proofs for a small open economy. We do not close the economy but assume that free international capital flows fix the domestic interest rate. The domestic wage is also endogenous.
} 


\section{The model}

\subsection{Optimal saving of one household}

Let an individual maximize a standard intertemporal utility function, $E_{t} \int_{t}^{\infty} e^{-\rho[\tau-t]} u(c(\tau)) d \tau$, where expectations need to be formed due to the uncertainty of labour income which in turn makes consumption $c(\tau)$ uncertain. The expectations operator is denoted $E_{t}$ and conditions on the current state in $t$. The planning horizon starts in $t$ and is infinite. The time preference rate $\rho$ is positive. We assume that the instantaneous utility functions has a CRRA structure

$$
u(c)=\frac{c^{1-\sigma}-1}{1-\sigma}, \quad \sigma>0, \sigma \neq 1 .
$$

Each individual can save and borrow in a riskless asset $a$ at interest rate $r>0$. The budget constraint of an individual reads

$$
d a(t)=\{r a(t)+z(t)-c(t)\} d t .
$$

Wealth $a(t)$ increases (or decreases) per unit of time $d t$ if capital income $r a(t)$ (where the interest rate $r$ is strictly positive) plus labour income $z(t)$ is larger (or smaller) than consumption $c(t)$. Labour income $z(t)$ jumps between two constants (which are determined further below). They could be called a real wage $w>b$ and an unemployment benefit $b>0$ or they could simply be seen as two wage levels. We understand $z(t)$ as a two-state Markov chain in continuous time with state space $\{w, b\}$, where the transition $w \rightarrow b$ happens with rate $s$ and the transition $b \rightarrow w$ with rate $\lambda$. Duration in states $w$ and $b$ is then exponentially distributed with mean $\lambda^{-1}$ and $s^{-1}$, respectively. This description of $z$ will be used in the remainder of the paper. As usual, the wealth-employment process $(a, z)$ is defined on a probability space $(\Omega, \mathcal{F}, P)$, equipped with the canonical filtration.

We now let the individual maximize her objective function by choosing a consumption function $c(a, z)$ subject to the budget constraint (2) and the Markov chain for the employment status. $^{7}$ In addition, we require that the individual holds wealth above the natural borrowing constraint (Aiyagari, 1994), i.e.

$$
a \geq-b / r .
$$

This implies that consumption when unemployed at the natural borrowing limit is zero,

$$
c(-b / r, b)=0 \text {. }
$$

The Bellman equation for this maximization problem reads ${ }^{8}$

$$
\rho V(a, z)=\max _{c(a, z)}\left\{\begin{array}{c}
\frac{c(a, z)^{1-\sigma}-1}{1-\sigma}+[r a+z-c(a, z)] V_{a}(a, z) \\
+\lambda[V(a, w)-V(a, z)]+s[V(a, b)-V(a, z)]
\end{array}\right\},
$$

where $V_{a}$ stands for the partial derivative of $V$ with respect to $a$. The first-order condition equates marginal utility from consumption with the shadow price of wealth,

$$
c(a, z)^{-\sigma}=V_{a}(a, z) .
$$

For our analysis to follow, we assume that the interest rate is lower than the time-preference rate, $r<\rho$. For convenience, we also assume that the initial wealth level $a(t)$ is chosen inside the interval $\left[-b / r, a_{w}^{*}\right]$. The lower bound $-b / r$ is the natural borrowing constraint from (3). The upper bound $a_{w}^{*}$ is endogenously determined further below. ${ }^{9}$

\footnotetext{
${ }^{7}$ There is no general theorem stating that optimal consumption is Markovian. We follow the economic tradition and restrict our attention to Markovian consumption functions.

${ }^{8}$ See Wälde (1999) or Keller et al. (2005) for related maximization problems with Bellman equations for exponentially distributed events.

${ }^{9}$ Our discussion below suggests that wealth will lie within this interval after a finite length of time with probability one even when initial wealth $a(t)$ lies above the intervall, i.e. for $a(t)>a_{w}^{*}$.
} 


\subsection{An illustration of consumption and wealth dynamics}

The dynamics of consumption and wealth can be illustrated in the wealth-consumption space. The background for this illustration results from initially focusing on the evolution between jumps (see Appendix B.1) and by eliminating time as exogenous variable. Computing the derivatives of consumption with respect to wealth in both states and considering wealth as the exogenous variable, we obtain (see Appendix A.1) a two-dimensional system of non-autonomous ordinary differential equations (ODE). The dynamics between jumps reads

$$
\begin{aligned}
\frac{d c(a, w)}{d a} & =\frac{r-\rho+s\left[\left(\frac{c(a, w)}{c(a, b)}\right)^{\sigma}-1\right]}{r a+w-c(a, w)} \frac{c(a, w)}{\sigma} \\
\frac{d c(a, b)}{d a} & =\frac{r-\rho-\lambda\left[1-\left(\frac{c(a, b)}{c(a, w)}\right)^{\sigma}\right]}{r a+b-c(a, b)} \frac{c(a, b)}{\sigma}
\end{aligned}
$$

With two boundary conditions, this system provides a unique solution for $c(a, w)$ and $c(a, b)$. These solutions are the deterministic part of our piece-wise deterministic system. The stochastic components result from the transitions at exponentially distributed points in time between employment and unemployment. The effect of a jump is then simply the effect of a jump of consumption from, say, $c(a, w)$ to $c(a, b)$.

The economics behind these equations is interesting. First, we see standard components like the budget constraints for both states in the denominators. We also see the usual determinants of consumption growth like the difference between the interest rate and the time preference rate, $r-\rho$, in both numerators and the measure of risk aversion $\sigma$. What this continuous time version of an optimal consumption rule nicely reveals are the determinants for precautionary saving (Leland, 1968, Aiyagari, 1994, Huggett and Ospina, 2001) in the good state - and those for dis-saving in the bad state. The term $s[\ldots]$ term in (7a) shows that consumption growth is faster under the risk of a job loss. Note that the expression $(c(a, w) / c(a, b))^{\sigma}-$ 1 is positive as consumption $c(a, b)$ of an unemployed worker is smaller than consumption of an employed worker $c(a, w)$ (see Lemma A.12 for a proof) and $\sigma>0$. When marginal utility from consumption under unemployment is much higher than marginal utility when employed, i.e. $(c(a, w) / c(a, b))^{\sigma}>>1$, individuals experience a high drop in consumption when becoming unemployed. As relative consumption shrinks when wealth rises, reducing this gap and smoothing consumption is best achieved by fast capital accumulation. This fast capital accumulation would go hand in hand with fast consumption growth as visible in (7a).

Similarly, the $\lambda[\ldots]$ term in (7b) shows that consumption growth for unemployed workers is smaller. The possibility to find a new job induces unemployed individuals to increase their current consumption level. Relative to a situation in which unemployment is an absorbing state (once unemployed, always unemployed, i.e. $\lambda=0$ ), the prospect of a higher labor income in the future reduces the willingness to give up today's consumption. With higher consumption levels, wealth accumulation is lower and consumption growth is reduced.

Properties of the system (7) can be illustrated in the usual way by plotting zero-motion lines and by plotting the sign of the derivatives into a phase diagram. Following these steps, it turns out (see Appendix A.2) that there is an endogenous upper limit $a_{w}^{*}$ of the wealth distribution determined by the zero-motion line for consumption. The ratio of consumption at this point is given by

$$
\left(\frac{c\left(a_{w}^{*}, w\right)}{c\left(a_{w}^{*}, b\right)}\right)^{\sigma}=1-\frac{r-\rho}{s} .
$$

Joint with the natural borrowing constraint (3), this allows us to plot a phase diagram as in Figure 1. This figure displays wealth on the horizontal and consumption $c(a, z)$ on the vertical axis. It plots dashed zero-motion lines for $a_{w}$, following from (2) for $z=w$, and for $c(a, w)$ 
and a solid zero-motion line for $a_{b} \cdot{ }^{10}$ We assume for this figure that the threshold level $a_{w}^{*}$ is positive. ${ }^{11}$ The intersection point of the zero-motion lines for $c(a, w)$ and $a_{w}$ is the temporary steady state (TSS),

$$
\Theta \equiv\left(a_{w}^{*}, c\left(a_{w}^{*}, w\right)\right)
$$

We call this point temporary steady state for two reasons. On the one hand, employed workers experience no change in wealth, consumption or any other variable when at this point (as in a standard steady state of a deterministic system). On the other hand, the expected spell in employment is finite and a random transition into unemployment will eventually occur. Hence, the state in $\Theta$ is steady only temporarily.

As we know from the proposition in Appendix A.2 that consumption for the unemployed always falls, both consumption and wealth fall above the zero-motion line for $a_{b}$. The arrowpairs for the employed workers are also added. They show that one can draw a saddle-path through the TSS. To the left of the TSS, wealth and consumption of employed workers rise, to the right, they fall.

Relative consumption when the employed worker is in the TSS is given by (8). A trajectory going through $\left(a_{w}^{*}, c\left(a_{w}^{*}, b\right)\right)$ and hitting the zero-motion line of $a_{b}$ at $-b / r$ is in accordance with laws of motions for the unemployed worker.

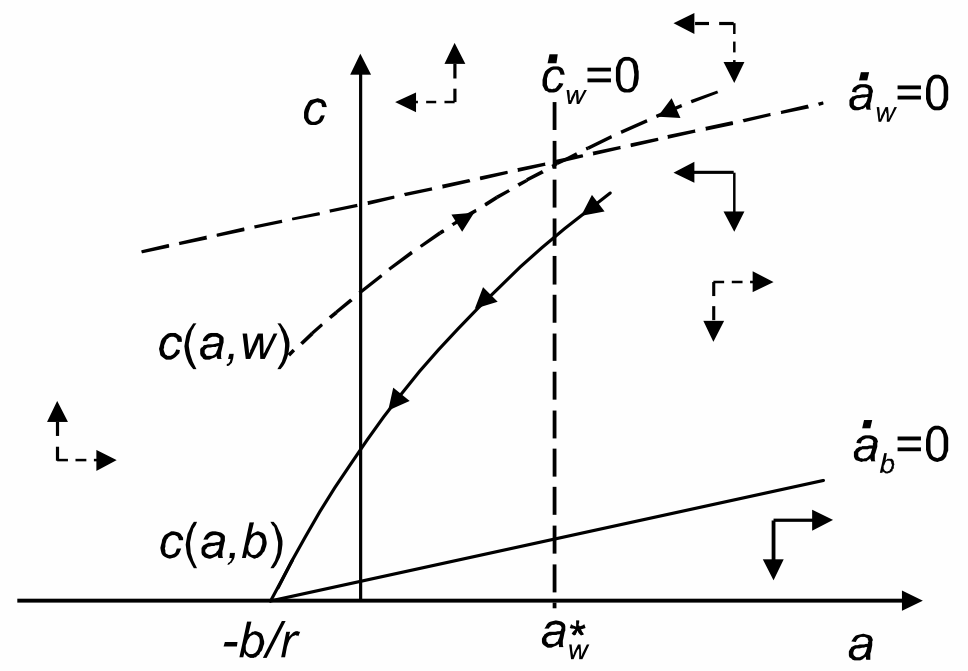

Figure 1 Policy functions for employed and unemployed workers

For our assumption of an interest rate being lower than the time preference rate, $r<\rho$, the range of wealth a worker can hold is bounded. Whatever the initial wealth level, there is a positive probability that the wealth level will be in the range $\left[-b / r, a_{w}^{*}\right]$ after some finite length of time. For an illustration, consider the policy functions in Figure 1: Wealth decreases both for employed and unemployed workers for $a>a_{w}^{*}$. The transition into the range $\left[-b / r, a_{w}^{*}\right]$ will take place only in the state of unemployment which, however, occurs with positive probability.

When wealth of an individual is within the range $\left[-b / r, a_{w}^{*}\right]$, consumption and wealth will rise while employed and fall while unemployed. While employed, precautionary saving motives drive the worker to accumulate wealth. While unemployed, the worker runs down current wealth as higher income for the future is anticipated - "postcautionary dis-saving" takes place. When a worker loses a job at a wealth level of, say, $a_{w}^{*} / 2$, his consumption level will drop

\footnotetext{
${ }^{10}$ Appendix A.2 and A.3 prove all properties of our system required for plotting this phase diagram.

${ }^{11}$ In an application explaining the evolution of the wealth distribution of the NLSY 79 cohort, Khieu and Wälde (2018) find positive values for the threshold for reasonable parameter values. The threshold approaches infinity for $r$ approaching $\rho$.
} 
from $c\left(a_{w}^{*} / 2, w\right)$ to $c\left(a_{w}^{*} / 2, b\right)$. Conversely, if an unemployed worker finds a job at, say, $a=0$, her consumption increases from $c(0, b)$ to $c(0, w)$. A worker will therefore be in a permanent consumption and wealth cycle. Given these dynamics, wealth will never leave the interval $\left[-b / r, a_{w}^{*}\right]$ and one can easily imagine a distribution of wealth over the range $\left[-b / r, a_{w}^{*}\right]$.

\subsection{Aggregation for a small open economy}

Having described one individual, we now consider an entire population with a very large number $N^{Y}$ of workers. Consider a small open economy that produces a final good $Y(t)$, employing capital $K(t)$ and labour $L(t)$. The final good is produced with a linearly homogenous production function with positive first and negative second derivatives. The labour force is fixed at $N^{Y}$ and the unemployment rate is $\left(N^{Y}-L(t)\right) / N^{Y}$. Firms act under perfect competition and there are free capital flows given a constant international interest rate $r$. This fixes the consumers' interest rate in our small open economy at $r$ as well. At invariant total factor productivity, a constant interest rate implies (via well-known properties of linearly homogenous functions) an invariant capital to labour ratio $K(t) / L(t)$ in this economy. When the unemployment rate changes, the capital stock per capita $K(t) / N^{Y}$ changes but the capital stock per worker $K(t) / L(t)$ remains constant. As a consequence, the real wage $w$ which, again by linear homogeneity of the production function, is a function of $K(t) / L(t)$ only, is a function of $r$ and thereby constant as well.

Unemployment benefits are determined as a fixed ratio (the replacement rate) of the wage and are financed by lump-sum taxes. There is a government budget constraint which is balanced at each point. As lump-sum taxes do not affect the maximization problem of individuals, we omit the tax term in the budget constraint (2) of the household.

Letting $N^{Y}$ be a continuum or letting it go to infinity with $N^{Y}$ being a discrete number, a standard law of large numbers implies that distributional properties we show in what follows apply to a population of a small open economy as well. When we show that the wealth distribution for some future point in time of one individual converges to a stationary distribution for this one individual, this implies at the same time that the cross-sectional distribution in an economy converges to the same stationary distribution.

\section{Existence and properties of the optimal consumption path}

This section provides a proof for the existence of a path $c(a, z)$ as depicted in Figure 1 and shows properties needed in our subsequent analysis of the distribution of wealth.

\subsection{Existence of the optimal consumption path}

In Figure 1, we implicitly considered solutions of our system in the set

$$
Q=\{a \geq-b / r\} \cap\{c(a, w) \leq r a+w\} \cap\{c(a, b) \geq r a+b\} \cap\{c(a, w) \geq c(a, b)\} .
$$

In words, wealth is at least as large as the maximum debt level $b / r$, consumption of the employed worker is below the zero-motion line for her wealth, consumption of the unemployed worker is above her zero-motion line for wealth (and thereby non-negative) and consumption of employed workers always exceeds consumption of unemployed workers (see Lemma A.12). We will show that the solution we construct indeed takes its values in the set $Q$.

In the following proofs we are faced with the problem that the coefficients in the equation (7) may become singular in some situations (i.e. at the bounds of $Q$ when $c(a, b)=0$ or $c(a, w)=$ 
$r a+w)$, preventing us from applying standard theorems. To overcome these difficulties, we regularize this equation (7) in certain ways by introducing small parameters. The regularized equation is easier to solve. Once this has been achieved, the additional parameters can be allowed to tend to zero in a controlled way, thus giving a solution of the original equation.

We start with a

Definition 4.1 (Optimal consumption path) A consumption path is a solution $(a, c(a, w), c(a, b))$ of the ODE-system (7) for the range $-b / r \leq a \leq a_{w}^{*}$ in $Q$ with terminal condition $\left(a_{w}^{*}, c\left(a_{w}^{*}, w\right), c\left(a_{w}^{*}, b\right)\right)$. Given the TSS from (9), the terminal condition satisfies

$$
c\left(a_{w}^{*}, w\right)=r a_{w}^{*}+w
$$

and $\left(c\left(a_{w}^{*}, w\right) / c\left(a_{w}^{*}, b\right)\right)^{\sigma}=1-\frac{r-\rho}{s}$ from (8) for an arbitrary $a_{w}^{*}>-b / r$. An optimal consumption path is a consumption path which in addition satisfies $c(-b / r, b)=0$ from (4).

To prove existence of an optimal consumption path, we proceed in steps. We first regularize the system by introducing a parameter $v$. This parameter is used to modify one of the conditions to be satisfied by the solution at the right endpoint of the interval of existence. This motivates the introduction of the modified domain

$$
Q_{v}=\left\{(a, c(a, w), c(a, b)) \in \mathbb{R}^{3} \mid(a, c(a, w), c(a, b)) \in Q, c(a, w) \leq r a+w-v\right\}
$$

where $v$ is a small positive constant, as an approximation to our "full" set $Q$. As $Q_{0}=Q$, $Q_{v}$ simply excludes the zero-motion line for wealth of the employed workers. We need to do this as the quotient on the right-hand side of our differential equation (7a) is not defined for the TSS. As $v$ is small, however, we can get arbitrarily close to this zero-motion line and $Q_{v}$ approximates $Q$ arbitrarily well. We then prove

Lemma 4.2 An optimal consumption path exists for $Q_{v}$ where the terminal condition is given by $\left(\hat{a}, c_{v}(\hat{a}, w), c_{v}(\hat{a}, b)\right)$ and where $c_{v}(\hat{a}, w)$ is defined in analogy to (11) as $c_{v}(\hat{a}, w)=r \hat{a}+w-v$ and $c_{v}(\hat{a}, b)$ is determined by $\left(c_{v}(\hat{a}, w) / c_{v}(\hat{a}, b)\right)^{\sigma}=1-\frac{r-\rho}{s}$ in analogy to (8).

Proof. see Appendix A.4

Building on this lemma, we can then prove

Theorem 4.3 An optimal consumption path exists for $Q$.

Proof. see Appendix A.5

\subsection{Properties of the consumption and wealth paths}

This section gathers some properties of the system needed for the stability analysis of the wealth distribution. As stated earlier, we assume that we are in the low-interest regime, $r<\rho$. We introduce the notation $\psi_{z}\left(a_{0}, t\right)$ for the wealth at time $t$ if the agent starts with initial wealth $a_{0}$ at time 0 and her employment status is constant at level $z(z \in\{w, b\})$. Optimally controlled wealth between jumps then follows

$$
\frac{\partial \psi_{z}\left(a_{0}, t\right)}{\partial t}=r \psi_{z}\left(a_{0}, t\right)+z-c\left(\psi_{z}\left(a_{0}, t\right), z\right), \quad \psi_{z}\left(a_{0}, 0\right)=a_{0}
$$

We can then formulate

Proposition 4.4 Properties of optimal consumption and the implied wealth path between jumps are as follows. 
(a) The map $a \mapsto c(a, z)$ is $C^{1}$ in the interior $]-b / r, a_{w}^{*}[$ of our system, $z=w, b$.

(b) $t \mapsto \psi_{z}\left(a_{0}, t\right)$ is increasing for $z=w$ and decreasing for $z=b$ and strictly so while $\psi_{w}\left(a_{0}, t\right)<a_{w}^{*}$ and $\psi_{b}\left(a_{0}, t\right)>-b / r$, respectively.

(c) $a_{0} \mapsto \psi_{z}\left(a_{0}, t\right)$ is continuous on $\left[-b / r, a_{w}^{*}\right]$,

(d) $\lim _{t \rightarrow \infty} \psi_{w}\left(a_{0}, t\right)=a_{w}^{*}, \lim _{t \rightarrow \infty} \psi_{b}\left(a_{0}, t\right)=-b / r$.

Proof. see Appendix A.6.

\section{$5 \quad$ Stability of the wealth-employment process}

We would now like to formally understand the stability properties of the model just presented. ${ }^{12}$ As the fundamental state variables are wealth (2) and the employment status $z(t)$ of an individual, the process we are interested in is the wealth-employment process $X_{\tau} \equiv(a(\tau), z(\tau))$. All other variables (like control variables or e.g. factor rewards in a general equilibrium version) are known deterministic functions of the state variables. Hence, if we understand the process governing the state variables, we also understand the properties of all other variables in this model. The state-space of this process $X_{\tau}$ is $\mathbf{X} \equiv\left[-b / r, a_{w}^{*}\right] \times\{w, b\}$ and has all the properties required for the state space in the general ergodicity theory for Markov processes, which we review in section 5.1 below. Moreover, for the sake of simplicity, we now set the initial time $t=0$ - following the usual practice in the mathematical literature.

The goal of this section is a proof of stability of the Markov process $X_{\tau}$ in the sense that we want to show that the distribution of $X_{\tau}$ converges for $\tau \rightarrow \infty$ to a unique limiting distribution (no matter what the initial value $X_{0}$ ). (See Definition 5.10 for the precise meaning of that statement.)

The general structure of the stability or ergodicity proof is quite usual:

- First we prove existence of an invariant probability measure, i.e., of a distribution $\mu$ on the state space such that the process is stationary when started with this distribution, i.e., when $X_{0} \sim \mu$. Hence, the first step is looking for candidates for the limiting distribution, if it exists. (Note that we here use "probability measure" and "distribution" essentially as synonyms.) As our state space is already compact, existence will follow from a continuity condition on the paths of $X$, more precisely the weak Feller property, cf. Definition 5.5 below. We review the theoretical underpinnings in section 5.1.2 and carry out the proofs for our model in section 5.2.

- Then we prove uniqueness of such invariant probability measures. Technically, the usual techniques actually only provide uniqueness of invariant measures (which may well be infinite if no invariant probability measure exists), but the combination with the first step, of course, gives existence and uniqueness of the invariant distribution. As in the case of Markov chains, uniqueness follows from irreducibility (Definition 5.1) and recurrence (Definition 5.3) of the process $X$. Proving the latter property requires us to have some smoothing properties of $X$, which is often easy to verify in a diffusion setting, but not so clear in a pure jump setting as ours. We critically rely on the notion of $T$-processes defined in Definition 5.8. Verifying that our wealth-employment process $X$ is a $T$-process is the main task of section 5.3.

\footnotetext{
${ }^{12}$ This proof circulated earlier in Bayer and Wälde (2010a,b).
} 
- The unique invariant distribution identified in the last step is the natural candidate for the limiting distribution, so we only have to prove convergence in the third step. This is done in section 5.4. Note that we are using the notion of convergence in total variation sense as compared to the more usual (and weaker) convergence in distribution.

We now continue with an overview of ergodicity theory for Markov processes in continuous time with continuous state spaces. All the results in section 5.1 are well known in the mathematical literature and, hence, the reader only interested in the new results might directly proceed with section 5.2.

\subsection{Review of ergodicity results for continuous-time Markov processes}

The wealth-employment process $(a(\tau), z(\tau))$ is a continuous-time Markov process with a nondiscrete state space $\left[-b / r, a_{w}^{*}\right] \times\{w, b\}$. Thus, we will rely on results from the general stability theory of Markov processes as presented in the works of Meyn and Tweedie and their coauthors cited above. In the present section, we will recapitulate the most important elements of the stability for Markov processes in continuous time. Here, we will discuss the theory in full generality, i.e., we assume that we are given a Markov process $\left(X_{t}\right)_{t \in \mathbb{R}_{>0}}$ on a state space $\mathbf{X}$, which is assumed to be a locally compact separable metric space endowed with its Borel $\sigma$-algebra. All Markov processes are assumed to be time-homogeneous, i.e., the conditional distribution of $X_{t+s}$ given $X_{t}=x$ only depends on $s$, not on $t .^{13}$

\subsubsection{Preliminaries}

Let $\left(X_{t}\right)_{t \in \mathbb{R}>0}$ be a (homogeneous) Markov process with the state space $\mathbf{X}$, where $\mathbf{X}$ is assumed to be a locally compact and separable metric space, which is endowed with its Borel $\sigma$-algebra $\mathcal{B}(\mathbf{X})$. Let $P^{t}(x, A), t \geq 0, x \in \mathbf{X}, A \in \mathcal{B}(\mathbf{X})$, denote the corresponding transition kernel, i.e.

$$
P^{t}(x, A) \equiv P\left(X_{t} \in A \mid X_{0}=x\right) \equiv P_{x}\left(X_{t} \in A\right),
$$

where $P_{x}$ is a shorthand-notation for the conditional probability $P\left(\cdot \mid X_{0}=x\right)$. Note that $P^{t}(\cdot, \cdot)$ is a Markov kernel, i.e. for every $x \in \mathbf{X}$, the map $A \mapsto P^{t}(x, A)$ is a probability measure on $\mathcal{B}(\mathbf{X})$ and for every $A \in \mathcal{B}(\mathbf{X})$, the map $x \mapsto P^{t}(x, A)$ is a measurable function. Similarly, by a kernel we understand a function $K:(\mathbf{X}, \mathcal{B}(\mathbf{X})) \rightarrow \mathbb{R}_{\geq 0}$ such that $K(x, \cdot)$ is a measure, not necessarily normed by 1 , for every $x$ and $K(\cdot, A)$ is a measurable function for every measurable set $A$. Moreover, let us denote the corresponding semi-group by $P_{t}$, i.e.

$$
P_{t} f(x) \equiv E\left(f\left(X_{t}\right) \mid X_{0}=x\right)=\int_{\mathbf{X}} f(y) P^{t}(x, d y)
$$

for $f: \mathbf{X} \rightarrow \mathbb{R}$ bounded measurable. For a measurable set $A$, we consider the stopping time $\tau_{A}$ and the number of visits of $X$ in set $A$,

$$
\tau_{A} \equiv \inf \left\{t \geq 0 \mid X_{t} \in A\right\}, \quad \eta_{A} \equiv \int_{0}^{\infty} \mathbf{1}_{A}\left(X_{t}\right) d t .
$$

Let us further recall that a measure $\varphi$ defined on $\mathcal{B}(\mathbf{X})$ is called $\sigma$-finite if we can decompose $\mathbf{X}$ into countably many measurable sets $A_{n}, n \in \mathbb{N}, \bigcup_{n \in \mathbb{N}} A_{n}=\mathbf{X}$, with $\varphi\left(A_{n}\right)<\infty$.

\footnotetext{
${ }^{13}$ All the results presented here are strongly linked with (discrete-time) Markov chains embedded in the continuous time Markov process. If one wanted to dig deeper, one would see that essentially all of the employed concepts can be related to concepts on the level of sampled discrete-time chains, be it irreducibility, recurrence, boundedness in probability or other. A truly comprehensive review of these connections would, however, go beyond the scope of this paper. We refer to Meyn and Tweedie (1993b) for a thorough account.
} 
Definition 5.1 Assume that there is a $\sigma$-finite, non-trivial measure $\varphi$ on $\mathcal{B}(\mathbf{X})$ such that, for sets $B \in \mathcal{B}(\mathbf{X}), \varphi(B)>0$ implies $E_{x}\left(\eta_{B}\right)>0, \forall x \in \mathbf{X}$. Here, similar to $P_{x}, E_{x}$ is a short-hand notation for the conditional expectation $E\left(\cdot \mid X_{0}=x\right)$. Then $X$ is called $\varphi$-irreducible.

In the more familiar case of a finite state space and discrete time, we would simply require $\eta_{\{x\}}$ to have positive expectation for any state $x$. In the continuous case, such a requirement would obviously be far too strong, since singletons $\{x\}$ usually have probability zero. The above definition only requires positive expectation for sets $B$, which are "large enough", in the sense that they are non-null for some reference measure.

A simple sufficient condition for irreducibility is given in Meyn and Tweedie (1993b, Proposition 2.1), which will be used to show irreducibility of the wealth-employment process.

Proposition 5.2 Suppose that there exists a $\sigma$-finite measure $\mu$ such that $\mu(B)>0$ implies that $P_{x}\left(\tau_{B}<\infty\right)>0$. Then $X$ is $\varphi$-irreducible, where

$$
\varphi(A) \equiv \int_{\mathbf{X}} R(x, A) \mu(d x), \quad R(x, A) \equiv \int_{0}^{\infty} P^{t}(x, A) e^{-t} d t
$$

We call $\varphi$ the irreducibility measure.

Definition 5.3 The process $X$ is called Harris recurrent if there is a non-trivial $\sigma$-finite measure $\varphi$ such that $\varphi(A)>0$ implies that $P_{x}\left(\eta_{A}=\infty\right)=1, \forall x \in \mathbf{X}$. Moreover, if a Harris recurrent process $X$ has an invariant probability measure, then it is called positive Harris.

Like in the discrete case, Harris recurrence may be equivalently defined by the existence of a $\sigma$-finite measure $\mu$ such that $\mu(A)>0$ implies that $P_{x}\left(\tau_{A}<\infty\right)=1$. As already remarked in the context of irreducibility, in the discrete framework one would consider sets $A=\{y\}$ with only one element.

Let $\mu$ be a measure on $(\mathbf{X}, \mathcal{B}(\mathbf{X}))$. We define a measure $P_{\mu}^{t}$ by

$$
P_{\mu}^{t}(A)=\int_{\mathbf{X}} P^{t}(x, A) \mu(d x) .
$$

We say that $\mu$ is an invariant measure, iff $P_{\mu}^{t}=\mu$ for all $t$. Here, the measure $\mu$ might be infinite. If it is a finite measure, we may, without loss of generality, normalize it to have total mass $\mu(\mathbf{X})=1$. The resulting probability measure is obviously still invariant, and we call it an invariant distribution. (Note that any constant multiple of an invariant measure is again invariant.) In the case of an invariant distribution, we can interpret invariance as meaning that the Markov process has always the same marginal distribution over time, when starting with the distribution $\mu$.

\subsubsection{Existence of an invariant probability measure}

The existence of finite invariant measures follows from a combination of two different types of conditions. The first property is a growth property. Several such properties have been used in the literature, a very useful one seems to be boundedness in probability on average.

Definition 5.4 The process $X$ is called bounded in probability on average if for every $x \in \mathbf{X}$ and every $\epsilon>0$ there is a compact set $C \subset \mathbf{X}$ such that

$$
\liminf _{t \rightarrow \infty} \frac{1}{t} \int_{0}^{t} P_{x}\left(X_{s} \in C\right) d s \geq 1-\epsilon
$$


The second property is a continuity condition.

Definition 5.5 The Markov process $X$ has the weak Feller property if for every continuous bounded function $f: \mathbf{X} \rightarrow \mathbb{R}$ the function $P_{t} f: \mathbf{X} \rightarrow \mathbb{R}$ from (15) is again continuous. Moreover, if $P_{t} f$ is continuous even for every bounded measurable function $f$, then $X$ has the strong Feller property.

Given these two conditions, Meyn and Tweedie (1993b, Theorem 3.1) establish the existence of an invariant probability measure in the following

Proposition 5.6 If a Markov process $X$ is bounded in probability on average and has the weak Feller property, then there is an invariant probability measure for $X$.

\subsubsection{Uniqueness}

Turning to uniqueness, the following proposition is cited in Meyn and Tweedie (1993b, page 491). For a proof see Azéma, Duflo and Revuz (1969, Théorème 2.5).

Proposition 5.7 If the Markov process $X$ is Harris recurrent and irreducible for a non-trivial $\sigma$-finite measure $\varphi$, then there is a unique invariant measure (up to constant multiples).

Proposition 5.7 gives existence and uniqueness of the invariant measure. A simple example shows that irreducibility and Harris recurrence do not guarantee existence of an invariant probability measure: Let $\mathbf{X}=\mathbb{R}$ and $X_{t}=B_{t}$ denote the one-dimensional Brownian motion. The Brownian motion is both irreducible and Harris recurrent - irreducibility is easily seen, while recurrence is well-known in dimension one (e.g. Karatzas and Shreve, 1988, Remark 6.20). Therefore, there is a unique invariant measure. By the Fokker-Planck equation, the density $f$ of the invariant measure must satisfy $f^{\prime \prime}=0$. By non-negativity, this implies that $f$ is constant, $f \equiv c$ for some $c>0 .{ }^{14}$ Thus, any invariant measure is a constant multiple of the Lebesgue measure, and there is no invariant probability measure for this example.

Given this example and as we are only interested in invariant probability measures, we need to combine this proposition with the previous section: Boundedness in probability on average together with the weak Feller property gives us the existence of an invariant probability measure as used in sect. 5.1.2, whereas irreducibility together with Harris recurrence imply uniqueness of invariant measures. Thus, for existence and uniqueness of the invariant probability measure, we will need all four conditions.

Whereas irreducibility, boundedness in probability on average and the weak Feller property are rather straightforward to check in practical situations, this seems to be harder for Harris recurrence. Thus, we next discuss some sufficient conditions for Harris recurrence. If the Markov process has the strong Feller property, then Harris recurrence will follow from a very weak growth property, namely that $P_{x}\left(X_{t} \rightarrow \infty\right)=0$ for all $x \in \mathbf{X}$, see Meyn and Tweedie (1993b, Theorem 3.2). Here, $X_{t} \rightarrow \infty$ is understood as: for any compact set $K$ there is a time $t_{0}$ s.t. $\forall t \geq t_{0}: X_{t} \in K^{c}$. In the case of a normed space, we might equivalently say that $\left\|X_{t}\right\| \rightarrow \infty$ for the relevant norm $\|\cdot\|$. While the strong Feller property is often satisfied for models driven by Brownian motion (e.g., for hypo-elliptic diffusions), it may not be satisfied in models where randomness is driven by a pure-jump process. Thus, we will next formulate an intermediate notion between the weak and strong Feller properties, which still guarantees enough smoothing for stability.

\footnotetext{
${ }^{14}$ See our earlier discussion papers Bayer and Wälde (2010a,b) for a heuristic derivation and analysis of Fokker-Planck equations for our model.
} 
Definition 5.8 The Markov process $X$ is called T-process, if there is a probability measure $\nu$ on $[0, \infty[$ and a kernel $T$ on $(\mathbf{X}, \mathcal{B}(\mathbf{X}))$ satisfying the following three conditions:

1. For every $A \in \mathcal{B}(\mathbf{X})$, the function $x \mapsto T(x, A)$ is continuous ${ }^{15}$.

2. For every $x \in \mathbf{X}$ and every $A \in \mathcal{B}(\mathbf{X})$ we have $K_{\nu}(x, A) \equiv \int_{0}^{\infty} P^{t}(x, A) \nu(d t) \geq T(x, A)$.

3. $T(x, \mathbf{X})>0$ for every $x \in \mathbf{X}$.

The kernel $K_{\nu}$ is the transition kernel of a discrete-time Markov process $\left(Y_{n}\right)_{n \in \mathbb{N}}$ obtained from $\left(X_{t}\right)_{t \geq 0}$ by random sampling according to the distribution $\nu$ : more precisely, let us draw a sequence $\sigma_{n}$ of independent samples from the distribution $\nu$ and define a discrete time process $Y_{n} \equiv X_{\sigma_{1}+\cdots+\sigma_{n}}, n \in \mathbb{N}$. Then the process $Y_{n}$ is Markov and has transition probabilities given by $K_{\nu}$. Using Definition 5.8 and Theorem 3.2 in Meyn and Tweedie (1993b), we can formulate

Proposition 5.9 Suppose that $X$ is a $\varphi$-irreducible T-process. Then it is Harris recurrent (with respect to $\varphi$ ) if and only if $P_{x}\left(X_{t} \rightarrow \infty\right)=0$ for every $x \in \mathbf{X}$.

Hence, in a practical sense and in order to prove existence of a unique invariant probability measure, one needs to establish that a process $X$ has the weak Feller property and is an irreducible $T$-process which is bounded in probability on average (as the latter implies the growth condition $P_{x}\left(X_{t} \rightarrow \infty\right)=0$ of Proposition 5.9).

Let us shortly compare the continuous, but compact case - where boundedness in probability is always satisfied - with the discrete and finite case. In the latter situation, existence of an invariant distribution always holds, while uniqueness is then given by irreducibility. In the compact, continuous case irreducibility and Harris recurrence only guarantee existence and uniqueness of an invariant measure, which might be infinite. On the other hand, existence of a finite invariant measure is given by the weak Feller property. Thus, for existence and uniqueness of an invariant probability measure, we will need the weak Feller property, irreducibility and Harris recurrence - which we will conclude from the T-property. Thus, the situation in the continuous (but compact) case is roughly the same as in the discrete case, except for some required continuity property, namely the weak Feller property.

\subsubsection{Stability}

By now we have established a framework for showing existence and uniqueness of an invariant distribution, i.e., probability measure. However, under stability we understand more, namely the convergence of the marginal distributions to the invariant distribution, i.e., that for any starting distribution $\mu$, the law $P_{\mu}^{\tau}$ of the Markov process at time $\tau$ converges to the unique invariant distribution for $\tau \rightarrow \infty$. In the context of $T$-processes, we are going to discuss two methods which allow to derive stability. But first, let us define the notion of stability in a more precise way.

Definition 5.10 For a signed measure $\mu$ consider the total variation norm

$$
\|\mu\| \equiv \sup _{|f| \leq 1}\left|\int_{\mathbf{X}} f(x) \mu(d x)\right|
$$

Then we call a Markov process $\left(X_{t}\right)_{t \in \mathbb{R}_{\geq 0}}$ stable or ergodic iff there is an invariant probability measure $\pi$ such that

$$
\forall x \in \mathbf{X}: \quad \lim _{t \rightarrow \infty}\left\|P^{t}(x, \cdot)-\pi\right\|=0 .
$$

\footnotetext{
${ }^{15} \mathrm{~A}$ more general definition requires lower semi-continuity only. As we can show continuity for our applications, we do not need this more general version here.
} 
Note that this implies in particular that the law $P_{\mu}^{t}$ of the Markov process converges to $\pi$, which is the unique invariant probability measure.

In the case of a finite state space in discrete time, ergodicity follows (inter alia) from aperiodicity. Down, Meyn and Tweedie (1995), also give one result for continuous state spaces in this direction.

Definition 5.11 A $\psi$-irreducible Markov process $\left(X_{t}\right)$ is called aperiodic iff there is a measurable set $C$ with $\psi(C)>0$ satisfying the following properties:

1. there is $\tau>0$ and a non-trivial measure $\nu$ on $\mathcal{B}(\mathbf{X})$ such that

$$
\forall x \in C, \forall A \in \mathcal{B}(\mathbf{X}): \quad P^{\tau}(x, A) \geq \nu(A) ;{ }^{16}
$$

2. there is $T>0$ such that

$$
\forall t \geq T, \forall x \in C: \quad P^{t}(x, C)>0 .
$$

If we are given an irreducible, aperiodic Markov process, then stability is implied by conditions on the infinitesimal generator. In the following proposition we give a special case of Down, Meyn and Tweedie (1995, Theorem 5.2) suitable for the employment-wealth process in our model.

Proposition 5.12 Given an irreducible, aperiodic T-process $X_{t}$ with infinitesimal generator $\mathcal{A}$ on a compact state space. Assume we can find a measurable function $V \in \mathcal{D}(\mathcal{A})$ with $V \geq 1$ and constants $d, c>0$ such that

$$
\mathcal{A} V \leq-c V+d
$$

Then the Markov-process is ergodic.

The problem with aperiodicity in the continuous-time framework is that it seems hard to characterize the small sets appearing in Definition 5.11. For this reason, we also give an alternative theorem, which avoids small sets (but is clearly related with the notion of aperiodicity).

Definition 5.13 Given a fixed $\tau>0$, the Markov process in discrete time $Y_{n} \equiv X_{\tau n}, n \in \mathbb{N}$ is called $a$ skeleton of $X$.

Meyn and Tweedie (1993b, Theorem 6.1) gives a characterization of stability in terms of irreducibility of skeleton chains.

Proposition 5.14 Given a Harris recurrent Markov process X with invariant probability measure $\pi$. Then $X$ is stable iff there is some irreducible skeleton chain.

\section{$5.2 \quad$ Existence}

After the review of the general ergodicity theory, we now come back and implement the scheme for our particular model. Hence, from now on we again work with the two-dimensional Markov process $X(\tau)=(a(\tau), z(\tau))$. As seen above, in order to show existence for an invariant probability measure for $X$, we need (i) some compactness result for $X$ like boundedness in probability on average recalled in Definition 5.4 and (ii) a continuity property like the weak Feller property, see Proposition 5.6. Showing that $X$ is bounded in probability on average is straightforward: According to Definition 5.4 we need to find a compact set for any initial condition $x$ and any small number $\epsilon$ such that the average probability to be in this set is larger than $1-\epsilon$. As our process $X_{\tau} \equiv(a(\tau), z(\tau))$ is bounded, we can choose the state-space $\mathbf{X} \equiv\left[-b / r, a_{w}^{*}\right] \times\{w, b\}$ as our set for any $x$ and $\epsilon$. Concerning the weak Feller property, we offer the following

\footnotetext{
${ }^{16}$ Such a set $C$ is then called small.
} 
Lemma 5.15 The wealth-employment process has the weak Feller property.

Proof. Let us first show that the wealth-employment process depends continuously on its initial values. To see this, fix some $\omega \in \Omega$, the probability space, on which the wealthemployment process is defined. Notice that $z_{\tau}(\omega)$ is certainly continuous in the starting values, because any function defined on $\{w, b\}$ is continuous by our choice of topology. Thus, we only need to consider the wealth process. For fixed $\omega, a_{\tau}(\omega)$ is a composition of solutions to deterministic ODEs, each of which are continuous functions of the respective initial value. Therefore, $a_{\tau}(\omega)$ is a continuous function of the initial wealth.

Now assume, without loss of generality, that the wealth-employment process has a deterministic initial value $\left(a_{0}, z_{0}\right)$ and fix some bounded, continuous function $f:\left[-b / r, a_{w}^{*}\right] \times\{w, b\} \rightarrow \mathbb{R}$. For the weak Feller property, we need to show that

$$
P_{\tau} f\left(a_{0}, z_{0}\right)=E\left(f\left(a_{\tau}, z_{\tau}\right)\right)
$$

is a continuous function in $\left(a_{0}, z_{0}\right)$. Thus, take any sequence $\left(a_{0}^{n}, z_{0}^{n}\right)$ converging to $\left(a_{0}, z_{0}\right)$ and denote the wealth-employment process started at $\left(a_{0}^{n}, z_{0}^{n}\right)$ by $\left(a_{\tau}^{n}, z_{\tau}^{n}\right)$. Then, by continuous dependence on the initial value, $\left(a_{\tau}^{n}(\omega), z_{\tau}^{n}(\omega)\right) \rightarrow\left(a_{\tau}(\omega), z_{\tau}(\omega)\right)$, for every $\omega \in \Omega$. By continuity of $f$, this implies convergence of $f\left(a_{\tau}^{n}(\omega), z_{\tau}^{n}(\omega)\right)$. Since $f$ is bounded, we may conclude convergence $P_{\tau} f\left(a_{0}^{n}, z_{0}^{n}\right) \rightarrow P_{\tau} f\left(a_{0}, z_{0}\right)$ by the dominated convergence theorem. Thus, $P_{\tau} f$ is, indeed, bounded and continuous whenever $f$ is bounded and continuous, and the weak Feller property holds.

\subsection{Uniqueness}

Given existence of an invariant distribution, uniqueness will follow from (Harris) recurrence together with irreducibility of the process $X$. The details are spelled out in section 5.1.3, in particular in Proposition 5.7.

\subsubsection{Irreducibility}

We prove irreducibility in the following

Lemma 5.16 In the low-interest-regime with $r<\rho,(a(\tau), z(\tau))$ is an irreducible Markov process, with the non-trivial irreducibility measure $\varphi$ introduced in Proposition 5.2.

Proof. Let $-b / r<a<a_{w}^{*}, z \in\{w, b\}$. Then, regardless of the initial point $a_{t} \in\left[-b / r, a_{w}^{*}\right]$ and regardless of $z_{t}$, it is possible to attain the state $(a, z)$ in finite time with probability greater than zero. Thus, Proposition 5.2 implies irreducibility with irreducibility measure

$$
\varphi(A) \equiv \int_{\mathbf{X}} R(x, A) \mu(d x), \quad R(x, A) \equiv \int_{0}^{\infty} P^{t}(x, A) e^{-t} d t
$$

where we can take the Lebesgue measure on $\left[-b / r, a_{w}^{*}\right]$ times the counting measure on $\{w, b\}$ as measure $\mu$.

\subsubsection{Harris recurrence}

The proof of Harris recurrence is more elaborate and builds on some auxiliary results, most importantly on being a $T$-process, compare Definition 5.8 which will be proved in Theorem 5.18 below. We start by giving an auxiliary result on the distribution of jumps in the employment status. 
Lemma 5.17 The conditional density of the time of the first jump in employment given that there is precisely one such jump in $[0, \tau]$ and that $z(0)=w$ is given by

$$
g_{\tau}^{(1)}(u)= \begin{cases}\frac{\lambda-s}{e^{(\lambda-s) \tau}-1} e^{(\lambda-s) u}, 0 \leq u \leq \tau, & \lambda \neq s \\ 1 / \tau, 0 \leq u \leq \tau, & \lambda=s .\end{cases}
$$

Proof. Since the formula is well-known for $\lambda=s$, we only prove the result for $\lambda \neq s$. The joint probability of the first jump $\tau_{1} \leq u \leq \tau$ and $N_{\tau}=1$, where $N_{\tau}$ denotes the number of jumps in $[0, \tau]$, is given by

$$
\begin{aligned}
P\left(\tau_{1}\right. & \left.\leq u, N_{\tau}=1\right)=P\left(\tau_{1} \leq u, \tau_{2} \geq \tau-\tau_{1}\right)=\int_{0}^{u} P\left(\tau_{2} \geq \tau-v\right) s e^{-s v} d v \\
& =\int_{0}^{u} e^{-\lambda(\tau-v)} s e^{-s v} d v=\frac{s}{\lambda-s} e^{-\lambda \tau}\left(e^{(\lambda-s) u}-1\right) .
\end{aligned}
$$

Here, $\tau_{2}$ denotes the time between the first and the second jump, and we have used independence of $\tau_{1}$ and $\tau_{2}$. Dividing through the probability of $N_{\tau}=1$, we get

$$
P\left(\tau_{1} \leq u \mid N_{\tau}=1\right)=\frac{e^{(\lambda-s) u}-1}{e^{(\lambda-s) t}-1},
$$

and we obtain the above density by differentiating with respect to $u$.

Before starting the somewhat elaborate proof of the $T$-property, let us shortly discuss why the conventional way to uniqueness of invariant measures is not open to us. As discussed in section 5.1, uniqueness of the invariant distribution of a Markov process is implied by smoothing properties of the process, and this approach is usually employed in the literature of continuoustime models. However, the wealth-employment process $(a, z)$ does not satisfy the strong Feller property (see Definition 5.5). Indeed, assume that $f:\left[-b / r, a_{w}^{*}\right] \times\{w, b\} \rightarrow \mathbb{R}$ is bounded measurable, but not continuous. For the sake of concreteness, let us assume that $f$ has a jump at some point $-b / r<a_{0}<a_{w}^{*}$. If there is no jump in the employment status until time $\tau$ (an event with positive probability), then the trajectory of the wealth process $a$ is deterministic until time $\tau$ and $z$ is even constant. Hence, on this event the jump cannot be smeared out.

On the other hand, the distribution of the jump times has a smooth density. If there is at least one jump until time $\tau$, we, therefore, expect the discontinuity of $f$ to be smeared out due to the density of the jump times. If both these heuristics are true, then

- the wealth-employment process is not strong Feller, as

$$
P_{\tau} f\left(a_{0}, z_{0}\right)=E\left[f\left(a_{\tau}, z_{\tau}\right)\right]=\underbrace{E\left[f\left(a_{\tau}, z_{\tau}\right) \mathbf{1}_{N_{\tau}=0}\right]}_{\text {discontinuous in }\left(a_{0}, z_{0}\right)}+\underbrace{E\left[f\left(a_{\tau}, z_{\tau}\right) \mathbf{1}_{N_{\tau}>0}\right]}_{\text {continuous in }\left(a_{0}, z_{0}\right)}
$$

is discontinuous in $\left(a_{0}, z_{0}\right)$ - where $N$ denotes the number of jumps in the employment status;

- the wealth-employment status conditioned on the number of jumps being greater then zero should satisfy the strong Feller condition. Hence, the kernel $T\left(\left(a_{0}, z_{0}\right), A\right)=P^{\tau}\left(\left(a_{0}, z_{0}\right), A \cap\right.$ $\left.\left\{N_{\tau}>0\right\}\right)$ should be a continuous component of $P^{\tau}$ in the sense of Definition 5.8. In other words, the wealth-employment process is a $T$-process.

Indeed, it turns out that these heuristic considerations lead to a correct conclusion.

Theorem 5.18 The wealth-employment process $(a(\tau), z(\tau))$ is a T-process. 
Given that there are some technical difficulties concerning the proof of Theorem 5.18, we first give a detailed heuristic sketch of the proof. A formal proof is provided afterwards. The main step in establishing that a kernel $T$ is a continuous component of $P^{\tau}$ in the sense of Definition 5.8 is to show continuity. To this end, let us consider a measurable set $A \subset\left[-b / r, a_{w}^{*}\right] \times\{w, b\}$ and define

$$
\begin{aligned}
& T_{>0}\left(\left(a_{0}, z_{0}\right), A\right) \equiv \int \mathbf{1}_{A}(a, w) p_{>0}^{\tau}\left(\left(a_{0}, z_{0}\right),(a, w)\right) d a P\left(N_{\tau}>0\right)+ \\
& \qquad \mathbf{1}_{A}(a, b) p_{>0}^{\tau}\left(\left(a_{0}, z_{0}\right),(a, b)\right) d a P\left(N_{\tau}>0\right),
\end{aligned}
$$

where $p_{>0}^{\tau}\left(\left(a_{0}, z_{0}\right),(a, z)\right)$ denotes the transition density of the wealth-employment process conditioned on $\left\{N_{\tau}>0\right\}$. That is, $T_{>0}$ describes the Markov dynamics on $\left\{N_{\tau}>0\right\}$. Obviously, continuity of $T_{>0}$ is equivalent to continuity of $a_{0} \mapsto p_{>0}^{\tau}\left(\left(a_{0}, z_{0}\right),(a, w)\right)$ and $a_{0} \mapsto$ $p_{>0}^{\tau}\left(\left(a_{0}, z_{0}\right),(a, b)\right)$. Moreover, if the heuristic argument is correct, we may actually restrict ourselves to the case when there is exactly one jump in the employment process until time $\tau$. This means, we consider the kernel

$$
T_{1}\left(\left(a_{0}, z_{0}\right), A\right) \equiv \int \mathbf{1}_{A}\left(a, z_{0}^{\prime}\right) p_{1}^{\tau}\left(\left(a_{0}, z_{0}\right),\left(a, z_{0}^{\prime}\right)\right) d a P\left(N_{\tau}=1\right)
$$

where $z_{0}^{\prime} \in\{w, b\}, z_{0}^{\prime} \neq z_{0}$ and $p_{1}^{\tau}$ denotes the transition density conditioned on the event that there is exactly one jump until time $\tau$. Similar to $T_{>0}, T_{1}$ describes the Markov dynamics on $\left\{N_{\tau}=1\right\}$. Now the picture becomes much clearer. Indeed, let us assume that the jump in employment status happens at some time $u<\tau$. Up to time $u$, the wealth process moves deterministically according to the ODE (2), after time $u$ it again moves in a deterministic way according to (2). Hence, there is a deterministic function $\phi_{z_{0}}$ (see (19) for the precise definition) such that

$$
a_{\tau}=\phi_{z_{0}}\left(a_{0}, u ; \tau\right)
$$

provided that there is precisely one jump of the employment status at time $u$ (and no other jump before $\tau$ ). Hence, we may express $T_{1}$ by

$$
T_{1}\left(\left(a_{0}, z_{0}\right), A\right)=\int_{0}^{\tau} \mathbf{1}_{A}\left(\phi_{z_{0}}\left(a_{0}, u ; \tau\right), z_{0}^{\prime}\right) g_{\tau}^{(1)}(u) d u P\left(N_{\tau}=1\right)
$$

If $u \mapsto \phi_{z_{0}}\left(a_{0}, u ; \tau\right)$ were smooth and invertible with smooth inverse $y \mapsto \phi_{z_{0}}^{-1}\left(a_{0}, y ; \tau\right)$, then we could re-write the equation as

$$
T_{1}\left(\left(a_{0}, z_{0}\right), A\right)=\int_{\operatorname{low}\left(a_{0}\right)}^{u p\left(a_{0}\right)} \mathbf{1}_{A}\left(y, z_{0}^{\prime}\right) g_{\tau}^{(1)}\left(\phi_{z_{0}}^{-1}\left(a_{0}, y ; \tau\right)\right)\left|\frac{\partial}{\partial y} \phi_{z_{0}}^{-1}\left(a_{0}, y ; \tau\right)\right| d y
$$

which is continuous in $a_{0}$ provided that $a_{0} \mapsto\left|\frac{\partial}{\partial y} \phi_{z_{0}}^{-1}\left(a_{0}, y ; \tau\right)\right|$ and $a_{0} \mapsto \operatorname{low}\left(a_{0}\right), a_{0} \mapsto u p\left(a_{0}\right)$ are continuous (plus some boundedness assumption). Assuming that we can make all these steps rigorous, we thus have proved the theorem.

In order to verify the various assumptions made in the above sketch, we need to understand the solution of the ODE

$$
\frac{d a_{z}(\tau)}{d \tau}=r a_{z}(\tau)+z-c\left(a_{z}(\tau), z\right)
$$

better. Indeed, the properties would be essentially trivial, if it were not for the (possible) singularity of the consumption function $c(a, z)$ at $a=-b / r$ and $a=a_{w}^{*}$ induced by the explosion of the right hand side in $(7)$. Nevertheless, by careful analysis we can establish the assumptions made above, at least when we further restrain the domain. 
We denote the solution of (18) started at $a_{0} \in\left[-b / r, a_{w}^{*}\right]$ at time 0 evaluated at time $\tau=u$ by $\psi_{z}\left(a_{0}, u\right)$, i.e., $\psi_{z}\left(a_{0}, 0\right)=a_{0}$. Let $\mathfrak{T}(a, z) \in[0, \infty]$ be the time it takes for the deterministic function $\psi_{z}(a, \cdot)$ to reach the boundary $\left\{-b / r, a_{w}^{*}\right\}$ of the domain. Note that $\mathfrak{T}$ may be infinite, which is actually the good situation, as the consumption function $c(a, z)$ is actually $C^{1}$ in that case -and, hence, stability holds. While it seems not clear how to obtain $C^{1}$ on the whole interval $\left[-b / r, a_{w}^{*}\right]$, it is clear how to get it on the interior of the domain, see Lemma 5.19. Of course, if $\mathfrak{T}(a, z)=\infty$ for some $a \in]-b / r, a_{w}^{*}[$, then it is infinite for any such $a$. Lemma 5.19 directly implies that $\psi_{z}(a, u)$ is $C^{1}$ in both $a$ and $u$ for $u<\mathfrak{T}(a, z)$, and continuous in both variables even for $u \leq \mathfrak{T}(a, z)$.

Lemma 5.19 The map $a \mapsto \mathfrak{T}(a, z)$ is continuous on $\left[-b / r, a_{w}^{*}\right] \backslash\left\{a_{z}^{*}\right\}$. Moreover, if $\mathfrak{T}(a, z)<$ $\infty$ for any $-b / r<a<a_{w}^{*}$, then $\mathfrak{T}(\cdot, z)$ is continuous on the whole domain. ${ }^{17}$

Proof. Let $\psi_{z}(a, u)$ denote the solution map of the ODE driving $a_{z}$ evaluated at time $u$ for initial value $\psi_{z}(a, 0)=a$. Obviously, $\psi_{w}(a, \cdot)$ is strictly increasing (until the time that $a_{w}^{*}$ is hit), while $\psi_{b}(a, \cdot)$ is strictly decreasing. Hence, they have continuous inverse functions (in $t$, for fixed $a$ ).

Fix any point $\left.a^{0} \in\right]-b / r, a_{w}^{*}\left[\right.$ and the corresponding value $\mathfrak{T}^{0}(z) \equiv \mathfrak{T}\left(a^{0}, z\right)$. For any positive $t$ we obviously have

$$
\mathfrak{T}\left(\psi_{z}(a, t), z\right)=\mathfrak{T}(a, z)-t .
$$

Denoting $\psi_{z}^{0}(t) \equiv \psi_{z}\left(a^{0}, t\right)$, we get for any $a<a^{0}$ for $z=b$ and any $a>a^{0}$ for $z=w$ that

$$
\mathfrak{T}(a, z)=\mathfrak{T}\left(\psi_{z}^{0}\left(\left(\psi_{z}^{0}\right)^{-1}(a)\right), z\right)=T^{0}(z)+\left(\psi_{z}^{0}\right)^{-1}(a),
$$

which is continuous in $a$. As $a^{0}$ was arbitrary in the interior of the interval, the claim follows.

Let us introduce a little bit of notation: for $z \in\{w, b\}$ we denote by $z^{\prime}$ the other element of $\{w, b\}$. Moreover, we define

$$
\phi_{z}(a, u ; \tau) \equiv \psi_{z^{\prime}}\left(\psi_{z}(a, u), \tau-u\right), \quad 0 \leq u \leq \tau, z \in\{w, b\}
$$

In words, $\phi_{z}$ denotes the value of the wealth process at time $\tau$ given that the wealth process at time 0 has the value $a$ and there is precisely one change of the employment status (from $z$ to $\left.z^{\prime}\right)$ in $[0, \tau]$, which takes place at time $u$. We are going to identify a sufficiently large set of $u$ s on which $u \mapsto \phi_{z}(a, u ; \tau)$ is differentiable and invertible with differentiable inverse.

Lemma 5.20 Define the set

$$
\mathfrak{S}(a, z ; \tau) \equiv\left\{u \in[0, \tau] \mid u>\tau-\mathfrak{T}\left(\psi_{z}(a, u), z^{\prime}\right)\right\}
$$

If $\mathfrak{T}\left(a, z^{\prime}\right)=\infty$ for some $-b / r<a<a_{w}^{*}$, i.e. when the boundary is never hit, then

$$
\mathfrak{S}(a, z ; \tau)= \begin{cases}{[0, \tau],} & a \neq a_{z^{\prime}}^{*} \\ ] 0, \tau], & a=a_{z^{\prime}}^{*}\end{cases}
$$

Otherwise, when a boundary is hit in finite time, the following three properties hold:

1. There are numbers $\mathfrak{s}(a, z ; \tau)$ such that $\mathfrak{S}(a, z ; \tau)=] \mathfrak{s}(a, z ; \tau), \tau]$.

2. $a \mapsto \mathfrak{s}(a, z ; \tau)$ is continuous on $]-b / r, a_{w}^{*}[$.

\footnotetext{
${ }^{17}$ Otherwise, we have a jump from $+\infty$ to 0 at $a=a_{z}^{*}$.
} 
3. For every $(a, z) \in\left[-b / r, a_{w}^{*}\right] \times\{w, b\}$ we have (uniformly) $\tau-\mathfrak{s}(a, z ; \tau)>0$.

Proof. The description for $\mathfrak{T}\left(a, z^{\prime}\right)=\infty$ is obvious, so we assume that $\forall a \in\left[-b / r, a_{w}^{*}\right] \backslash$ $\left\{a_{z^{\prime}}^{*}\right\}: \mathfrak{T}\left(a, z^{\prime}\right)<\infty$.

First note that $\tau \in \mathfrak{S}(a, z ; \tau)$. Moreover, for $u<v<\tau$ we have that $u \in \mathfrak{S}(a, z ; \tau)$ implies $v \in \mathfrak{S}(a, z ; \tau)$, since

$$
\tau-\mathfrak{T}\left(\psi_{z}(a, v), z^{\prime}\right) \leq \tau-\mathfrak{T}\left(\psi_{z}(a, u), z^{\prime}\right)<u<v,
$$

which shows that $\mathfrak{S}(a, z ; \tau)$ is an interval. However, for its lower endpoint the inequality is no longer strict, implying that the interval is closed to the right, but open to the left.

For the continuity of $\mathfrak{s}$, let us consider any (monotone) converging sequence $a_{n} \rightarrow a \in$ $\left[-b / r, a_{w}^{*}\right]$. First, assume that $u \in \mathfrak{S}\left(a_{n}, z ; \tau\right)$ for all $n \geq \bar{N}$, where $\bar{N}$ is a natural number. Then $u>\tau-\mathfrak{T}\left(\psi_{z}\left(a_{n}, u\right), z^{\prime}\right)$. Thus, continuity of $\psi_{z}(\cdot, u)$ and $\mathfrak{T}\left(\cdot, z^{\prime}\right)$ (cf. Lemma 5.19) imply that

$$
u \geq \tau-\mathfrak{T}\left(\psi_{z}(a, u), z^{\prime}\right) .
$$

The right hand side of the inequality is decreasing in $u$, so that we can infer that every $u^{\prime}>u$ is contained in $\mathfrak{S}(a, z ; \tau)$, hence $u \in \overline{\mathfrak{S}(a, z ; \tau)}$. In a similar way, we can show that $u \in$ $[0, \tau] \backslash \overline{\mathfrak{S}\left(a_{n}, z ; \tau\right)}$ for every $n \geq \bar{N}$ implies that $u \in[0, \tau] \backslash \mathfrak{S}(a, z ; \tau)$. However, this is only possible if $\mathfrak{s}\left(a_{n}, z ; \tau\right) \rightarrow \mathfrak{s}(a, z ; \tau)$, proving continuity in the interior of the domain.

It is obvious that $\tau>\mathfrak{s}(a, z ; \tau)$ as $\tau \in \mathfrak{S}(a, z ; \tau)$ and $\mathfrak{S}(a, z ; \tau)$ is half-open. The uniformity is also clear.

Lemma 5.21 The map $u \mapsto \phi_{z}(a, u ; \tau)$ is differentiable on $\mathfrak{S}(a, z ; \tau)$ and we have

$$
\left|\frac{\partial}{\partial u} \phi_{z}(a, u ; \tau)\right|>0 .
$$

Proof. By (19), $\phi_{z}$ is differentiable in $u$ provided that $a^{\prime} \mapsto \psi_{z^{\prime}}\left(a^{\prime}, \tau-u\right)$ is differentiable at $a^{\prime}=\psi_{z}(a, u)$. It is a well-known fact that the solution map of an ODE is differentiable in its initial value provided that the right hand side is $C^{1}$. By Proposition 4.4, the right hand side of (18) (for $z=z^{\prime}$ ) is $C^{1}$ (in $a$ ) as long as we do not hit $a_{z^{\prime}}^{*}$, which is precisely guaranteed by $u \in \mathfrak{S}(a, z ; \tau)$. Hence, we can apply the chain rule and obtain

$$
\begin{aligned}
\frac{\partial}{\partial u} \phi_{z}(a, u ; \tau)= & -\frac{\partial \psi_{z^{\prime}}}{\partial u}\left(\psi_{z}(a, u), \tau-u\right)+\frac{\partial \psi_{z^{\prime}}}{\partial a}\left(\psi_{z}(a, u), \tau-u\right) \frac{\partial \psi_{z}}{\partial u}(a, u) \\
= & -\underbrace{\left[r \phi_{z}(a, u ; \tau)+z^{\prime}-c\left(\phi_{z}(a, u, \tau), z^{\prime}\right)\right]}_{I}+ \\
& +\underbrace{\frac{\partial \psi_{z^{\prime}}}{\partial a}\left(\psi_{z}(a, u), \tau-u\right)}_{I I} \underbrace{\left[r \psi_{z}(a, u)+z-c\left(\psi_{z}(a, u), z\right)\right]}_{I I I} .
\end{aligned}
$$

For $z=w$, we have $I<0$ (with strict inequality as $u \in \mathfrak{S}(a, z ; \tau)$ ), and $I I \geq 0, I I I \geq 0$, implying that

$$
\frac{\partial}{\partial u} \phi_{w}(a, u ; \tau)>0
$$

On the other hand, for $z=b$, we have $I>0$ (again, with strict inequality), $I I \geq 0$ and $I I I \leq 0$, implying that

$$
\frac{\partial}{\partial u} \phi_{b}(a, u ; \tau)<0 .
$$


By Lemma 5.21 together with Lemma 5.20 we now understand rigorously on which domains of integration we can do the change of variables in (17), which is crucial for establishing continuity. Therefore, we are now prepared to finish the proof of the theorem.

Proof of th. 5.18. We choose the measure $\nu(d t)=\delta_{\tau}(d t)$ for some fixed $\tau>0$ and define a candidate $\widetilde{T}$ for a continuous component of $P^{\tau}$ by

$$
\widetilde{T}((a, z), A) \equiv \int_{0}^{\tau} \mathbf{1}_{A}\left(\phi_{z}(a, u ; \tau), z^{\prime}\right) \mathbf{1}_{\mathfrak{S}(a, z ; \tau)}\left(\phi_{z}(a, u ; \tau)\right) g_{\tau}^{(1)}(u) d u P\left(N_{\tau}=1\right),
$$

for $a \in\left[-b / r, a_{w}^{*}\right], z \in\{w, b\}, A \subset\left[-b / r, a_{w}^{*}\right] \times\{w, b\}$ measurable, i.e., $\widetilde{T}$ describes the dynamic of the Markov system on the event that the number of jumps $N_{\tau}$ of the system before time $\tau$ is equal to 1 such that the corresponding jump time $T_{1} \in \mathfrak{S}(a, z ; \tau)$. Hence, it is clear that $\widetilde{T} \leq P^{\tau}$. Now, introduce a change or variables $u \rightarrow y \equiv \phi_{z}(a, y ; \tau)$ as in (17). By Lemma 5.21, we get

$$
\begin{gathered}
\widetilde{T}((a, z), A)=\int_{L(a, z ; \tau)}^{U(a, z ; \tau)} \mathbf{1}_{A}\left(y, z^{\prime}\right) \mathbf{1}_{\mathfrak{S}(a, z ; \tau)}\left(\phi_{z}^{-1}(a, y ; \tau)\right) \times \cdots \\
\cdots \times g_{\tau}^{(1)}\left(\phi_{z}^{-1}(a, y ; \tau)\right)\left|\frac{\partial}{\partial y} \phi_{z}^{-1}(a, y ; \tau)\right| d y
\end{gathered}
$$

where the lower and upper limits of the integration are given by

$$
L(a, z ; \tau) \equiv\left\{\begin{array} { l l } 
{ \phi _ { z } ( a , 0 ; \tau ) , } & { z = w , } \\
{ \phi _ { z } ( a , \tau ; \tau ) , } & { z = b , }
\end{array} \quad U ( a , z ; \tau ) \equiv \left\{\begin{array}{ll}
\phi_{z}(a, \tau ; \tau), & z=w \\
\phi_{z}(a, 0 ; \tau), & z=b
\end{array}\right.\right.
$$

respectively. Here, $y \mapsto \phi_{z}^{-1}(a, y ; \tau)$ denotes the inverse function of $u \mapsto \phi_{z}(a, u ; \tau)$. Comparing (21) with (20), we note two important differences: the integrand (including the limits of the integration) in (21) is continuous in $a$ almost everywhere but, on the other hand, generally unbounded.

By a slight abuse of notation, let us denote $\mathfrak{S}(a, z ; \tau) \equiv] \mathfrak{s}(a, z ; \tau), \tau]{ }^{18}$ Lemma 5.20 implies that we may choose $0<\epsilon<\inf _{(a, z)}(\tau-\mathfrak{s}(a, z ; \tau))$. Now define $\left.\left.\mathfrak{S}_{\epsilon}(a, z ; \tau) \equiv\right] \mathfrak{s}(a, z ; \tau)+\epsilon, \tau\right]$ and

$$
T((a, z), A) \equiv \int_{0}^{\tau} \mathbf{1}_{A}\left(\phi_{z}(a, u ; \tau), z^{\prime}\right) \mathbf{1}_{\mathfrak{S}_{\epsilon}(a, z ; \tau)}\left(\phi_{z}(a, u ; \tau)\right) g_{\tau}^{(1)}(u) d u P(N(\tau)=1) .
$$

By the same change of variables as above, we arrive at

$$
\begin{gathered}
T((a, z), A)=\int_{L(a, z ; \tau)}^{U(a, z ; \tau)} \mathbf{1}_{A}\left(y, z^{\prime}\right) \mathbf{1}_{\mathfrak{S}_{\epsilon}(a, z ; \tau)}\left(\phi_{z}^{-1}(a, y ; \tau)\right) \times \cdots \\
\cdots \times g_{\tau}^{(1)}\left(\phi_{z}^{-1}(a, y ; \tau)\right)\left|\frac{\partial}{\partial y} \phi_{z}^{-1}(a, y ; \tau)\right| d y .
\end{gathered}
$$

Since the term $I$ in the proof of Lemma 5.21 only gets close to 0 when $u$ is close to $\mathfrak{s}(a, z ; \tau)$, now

$$
\mathbf{1}_{\mathfrak{S}_{\epsilon}(a, z ; \tau)}\left(\phi_{z}^{-1}(a, y ; \tau)\right)\left|\frac{\partial}{\partial y} \phi_{z}^{-1}(a, y ; \tau)\right|
$$

is uniformly bounded, implying that $(a, z) \mapsto T((a, z), A)$ is continuous for any measurable set A.

As, by construction, $\tau-\left(\mathfrak{s}\left(a_{0}, z_{0} ; \tau\right)+\epsilon\right)>0$ we have $T\left((a, z),\left[-b / r, a_{w}^{*}\right] \times\{w, b\}\right)>0$. Finally, it is obvious that $T((a, z), A) \leq \widetilde{T}((a, z), A) \leq P^{\tau}((a, z), A)$ for any $(a, z)$ and any measurable function $A$.

\footnotetext{
${ }^{18}$ This means that $\mathfrak{s}(a, z ; \tau) \equiv 0$ in the case $\mathfrak{T}\left(a, z^{\prime}\right)=\infty$ and $\mathfrak{S}(a, z ; \tau)=[0, \tau]$ is replaced by $\left.] 0, \tau\right]$ in that case.
} 
Corollary 5.22 The wealth-employment process $(a(\tau), z(\tau))$ is Harris recurrent.

Proof. By Lemma 5.16 and Theorem 5.18, the employment-wealth process $(a(\tau), z(\tau))$ is an irreducible $T$-process. Thus, Proposition 5.9 implies that $(a(\tau), z(\tau))$ is Harris recurrent, given that $P_{x}\left(X_{t} \rightarrow \infty\right)=0$ holds for our bounded state space.

\subsubsection{Uniqueness}

We can now complete our proof of uniqueness.

Theorem 5.23 Suppose that $r<\rho$. Then there is a unique invariant probability measure for the wealth-employment process $(a(\tau), z(\tau))$.

Proof. By Proposition 5.7, there is a unique invariant measure (up to a constant multiplier), and Proposition 5.6 implies that we may choose the invariant measure to be a probability measure.

\subsection{Stability}

Stability, i.e., convergence of the distribution of $(a(\tau), z(\tau))$ to the unique invariant distribution for any given initial distribution is implied by the existence of an irreducible skeleton chain, see Proposition 5.14.

Corollary 5.24 Under the assumptions of Theorem 5.23, the employment-wealth process is stable in the sense of Definition 5.10 .

Proof. Recall that the employment-wealth-process is a $T$-process, see Theorem 5.18. Moreover, we have shown irreducibility in Lemma 5.16. Proposition 5.14 will imply the desired conclusion, if we can show irreducibility of a skeleton chain. Take any $\tau>0$ and consider the corresponding skeleton $Y_{n}, n \in \mathbb{N}$, with transition probabilities $P^{\tau}$. By the proof of Theorem 5.18, we see that $\left(Y_{n}\right)$ is also a $T$-process, where the definition of $T$-processes is generalized to discrete-time processes in the obvious way. By Meyn and Tweedie (1993, Proposition 6.2.1), the discrete-time $T$-process $Y$ is irreducible if there is a point $x \in \mathbf{X}$ such that for any open neighborhood $O$ of $x$, we have

$$
\forall y \in \mathbf{X}: \quad \sum_{n=1}^{\infty} P^{n \tau}(y, O)>0 .
$$

This property, however, can be easily shown for the wealth-employment process $(a, z)$ as illustrated in Figure 1 and formally analysed in Appendix A.3.1 and 4. Indeed, take $x=(-b / r, b)$. Then any open neighborhood $O$ of $x$ contains $[-b / r,-b / r+\epsilon[\times\{b\}$ for some $\epsilon>0$. We start at some point $y=\left(a_{0}, z_{0}\right) \in \mathbf{X}$ and assume the following scenario: if necessary, at some time between 0 and $\tau$, the employment status changes to $b$, then it stays constant until the random time $N \tau$ defined by $N \equiv \inf \{n \mid a(n \tau)<-b / r+\epsilon\}$. Note that the wealth is decreasing in a deterministic way while $z=b$. Thus, we can find a deterministic upper bound $N \leq K\left(a_{0}\right)$. The event that the employment attains the value $b$ during the time interval $[0, \tau]$ and retains this value until time $K\left(a_{0}\right) \tau$ has positive probability. In this case, however, the trajectory of the wealth-employment process reaches $O$, implying that $\sum_{n=1}^{\infty} P^{n \tau}(y, O)>0$. Thus, the $\tau$-skeleton chain is irreducible and the wealth-employment process is stable. 


\section{Conclusion}

This paper has introduced methods that allow us to prove existence of an optimal consumption path that results from a setup with continuous time uncertainty. The fundamental stochastic process is a two-state Markov chain for labour income. Consumption and wealth accumulation is chosen optimally in our model of precautionary saving. We proved existence of a Markov consumption being a function of wealth and labour income. We exploited the fact that in our piecewise continuous system, the behaviour of optimal consumption between jumps can be described by an ODE system.

We also proved existence, uniqueness and stability of distributions resulting from this system. The distributions are a distribution of (optimally controlled) wealth and a distribution for the labour market status. The results hold for an interest rate being lower than the timepreference rate. By a standard law of large numbers, our findings also hold for the wealth and employment dynamics of any population and also, as in our interpretation, for a population of a small open economy.

The $T$-property turned out to be especially useful for models where randomness is introduced by finite-activity jump processes, i.e., by compound Poisson processes. In diffusion models, usually even the strong Feller property holds, which makes it easy to conclude the $T$-property. On the other hand, in models driven by infinite-activity jump processes, e.g., Lévy processes with infinite activity, it does not seem clear whether the $T$-property can lead to useful results. Indeed, in these models, the strong Feller property may and may not hold, see, for instance, Picard (1995/97). On the other hand, the weak Feller property is satisfied for all Lévy processes, implying existence of invariant distributions, see Applebaum (2004, Theorem 3.1.9). Looking at these issues in economic applications offers many fascinating research projects for years to come.

\section{A Appendix}

This section provides all properties of our system required for the phase diagram in Figure 1.

\section{A.1 Obtaining the system of ODEs for consumption}

When we derive the Keynes-Ramsey rules starting from the Bellman equation (5) and the first-order condition (6) in the usual way (see Appendix B.1), we obtain the following system consisting of two Keynes-Ramsey rules and two budget constraints. Optimal consumption when employed follows

$$
\frac{d c\left(a_{w}(t), w\right)}{c\left(a_{w}(t), w\right)}=\left\{\frac{r-\rho}{\sigma}+\frac{s}{\sigma}\left[\left(\frac{c\left(a_{w}(t), w\right)}{c\left(a_{w}(t), b\right)}\right)^{\sigma}-1\right]\right\} d t+\left[\frac{c\left(a_{w}(t), b\right)}{c\left(a_{w}(t), w\right)}-1\right] d q_{s}(t)
$$

while wealth evolves according to

$$
d a_{w}(t)=\left[r a_{w}(t)+w-c\left(a_{w}(t), w\right)\right] d t
$$

Optimal consumption when unemployed follows

$$
\frac{d c\left(a_{b}(t), b\right)}{c\left(a_{b}(t), b\right)}=\left\{\frac{r-\rho}{\sigma}-\frac{\lambda}{\sigma}\left[1-\left(\frac{c\left(a_{b}(t), b\right)}{c\left(a_{b}(t), w\right)}\right)^{\sigma}\right]\right\} d t+\left[\frac{c\left(a_{b}(t), w\right)}{c\left(a_{b}(t), b\right)}-1\right] d q_{\lambda}(t)
$$

and wealth follows

$$
d a_{b}(t)=\left[r a_{b}(t)+b-c\left(a_{b}(t), b\right)\right] d t
$$


Note that we apply an index to the state variable $a(t)$. Imagine, we had denoted the state variable wealth by one variable $a(t)$ in both states. Then the budget constraints would read $d a(t)=[r a(t)+w-c(a(t), w)] d t$ and $d a(t)=[r a(t)+b-c(a(t), b)] d t$. This might tempt to conclude that $w-c(a(t), w)=b-c(a(t), b)$ at each point even though one budget constraint holds for one state and the other for the other such that an individual can never be in both states at the same time. To avoid this, we index the single state variable $a(t)$ by $z$ to emphasize for which state wealth is being studied.

This system is piece-wise continuous and our discussion of Figure 1 will distinguish times between jumps and the effect of a transition from one labour market state to another. When we study these equations for the time between jumps, we set $d q_{s}=d q_{\lambda}=0$. We can eliminate time by computing $d c\left(a_{w}, w\right) / d a_{w}$ and $d c\left(a_{b}, b\right) / d a_{b}$ and replace $a_{w}$ and $a_{b}$ by $a .{ }^{19}$ We can do the latter as we now study the derivative of the consumption function with respect to wealth. Here, the time component no longer plays a role and the "risk" of comparing wealth levels in different states is no longer present. Going through these steps yields (7) in the main text. The stochastic $d q$-terms in (A.1a) and (A.1c) (tautologically) represent the discrete jumps in the level of consumption whenever the employment status changes.

\section{A.2 A proposition on consumption growth}

We focus on individuals in periods between jumps. Understanding the effects of jumps subsequently is trivial. The evolution of consumption between jumps is given by the deterministic part, i.e. the $d t$-part, in (A.1a) and (A.1c). We then easily understand

Lemma A.1 Individual consumption rises if and only if current consumption relative to consumption in the other state is sufficiently high.

For the employed worker, consumption rises if and only if $c\left(a_{w}, w\right)$ relative to $c\left(a_{w}, b\right)$ is sufficiently high,

$$
\frac{d c\left(a_{w}, w\right)}{d t} \geq 0 \Leftrightarrow\left(\frac{c\left(a_{w}, w\right)}{c\left(a_{w}, b\right)}\right)^{\sigma} \geq 1-\frac{r-\rho}{s} \Leftrightarrow \frac{c\left(a_{w}, w\right)}{c\left(a_{w}, b\right)} \geq 1 / \psi,
$$

where

$$
\psi \equiv\left(1-\frac{r-\rho}{s}\right)^{-1 / \sigma}
$$

For the unemployed worker, consumption rises if and only if $c\left(a_{b}, b\right)$ relative to $c\left(a_{b}, w\right)$ is sufficiently high,

$$
\frac{d c\left(a_{b}, b\right)}{d t} \geq 0 \Leftrightarrow\left(\frac{c\left(a_{b}, b\right)}{c\left(a_{b}, w\right)}\right)^{\sigma} \geq 1-\frac{r-\rho}{\lambda} \Leftrightarrow \frac{c\left(a_{b}, b\right)}{c\left(a_{b}, w\right)} \geq\left(1-\frac{r-\rho}{\lambda}\right)^{1 / \sigma} .
$$

Proof. Rearranging (A.1a) and (A.1c) for $d q_{s}=d q_{\lambda}=0$ gives the results.

We rely on the following lemma for our proposition below. It reads

Lemma A.2 Relative consumption $c(a, w) / c(a, b)$ is continuously differentiable in wealth a.

Proof. Consumption levels $c(a, w)$ and $c(a, b)$ are understood as solutions to our ODE system (7). As the latter is well-behaved within the set $Q_{v}$ from (12), consumption levels are continuously differentiable in $Q_{v}$. This implies that $c(a, w) / c(a, b)$ is also continuously differentiable.

\footnotetext{
${ }^{19}$ Eliminating time as exogenous variable means, simply speaking, to consider one ODE $d y(x) / d x=$ $f(y(x), x) / g(y(x), x)$ instead of considering two ODEs of the type $d y(t) / d t=f(y(t), x(t))$ and $d x(t) / d t=$ $g(y(t), x(t))$.
} 
For what follows, it is important to strengthen the statement of Lemma A.2. Suppose that $I$ is an open interval of the real line. A real-valued function $f$ on $I$ is called analytic if the following condition holds (see e.g. Krantz and Parks, 2002). For any fixed $x_{0} \in I$ there exists an $\epsilon>0$ and a sequence of real numbers $a_{i}$ such that $\left(x_{0}-\epsilon, x_{0}+\epsilon\right) \subset I$ and the series $\sum_{i=0}^{\infty} a_{i} x^{i}$ converges uniformly to $f$ on $\left(x_{0}-\epsilon, x_{0}+\epsilon\right)$. In other words, the function $f$ has a convergent power series expansion on a neighbourhood of $x_{0}$. Analytic functions of several variables can be defined in an analogous way. The usual elementary functions such as powers are analytic wherever they are differentiable. Sums, products, quotients (excluding division by zero) and compositions of analytic functions are analytic (Krantz and Parks, 2002, Chapter 1). As long as the unknowns remain in the set $Q_{v}$ the coefficients of the differential equations for $x$ and $y$ are analytic. It follows that the solutions are also analytic by the theorem of Cauchy-Kovalevskaya (Krantz and Parks, 2002, Chapter 1). Thus their quotient is analytic. Suppose now that $f$ is an analytic function on an open interval $I$ and $J \subset I$ is a closed interval. Then unless $f$ is identically zero the number of zeroes of $f$ in $J$ is finite (Krantz and Parks, 2002, Chapter 1). This allows us to formulate

Lemma A.3 The number of sign changes of the derivative of relative consumption with respect to wealth, i.e. $d(c(a, w) / c(a, b)) / d a$, in any interval of finite length is finite.

Proof. The derivative of an analytic function is analytic and so we can apply the above discussion, choosing the function $f$ to be $d(c(a, w) / c(a, b)) / d a$.

Proposition A.4 Consider a low interest rate, i.e. $0<r \leq \rho$. Define a threshold level $a_{w}^{*}$ by

$$
\frac{u^{\prime}\left(c\left(a_{w}^{*}, b\right)\right)}{u^{\prime}\left(c\left(a_{w}^{*}, w\right)\right)} \equiv 1-\frac{r-\rho}{s}
$$

For our instantaneous utility function (1), this definition reads

$$
c\left(a_{w}^{*}, b\right)=\psi c\left(a_{w}^{*}, w\right)
$$

where $\psi$ is from (A.3).

(i) Consumption of employed workers increases if the worker owns a sufficiently low wealth level, $a<a_{w}^{*}$. Employed workers with $a>a_{w}^{*}$ choose falling consumption paths.

(ii) Consumption of unemployed workers always decreases.

(iii) Consumption of employed workers exceeds consumption of unemployed workers at the threshold $a_{w}^{*}$, i.e. $\psi \leq 1$ in (A.6) for $r \leq \rho$.

Proof. see Appendix A.3

\section{A.3 Proof of the proposition on consumption growth}

\section{A.3.1 Proof of part (i)}

- A local result $a_{w}^{*}$.

We first show that consumption $c\left(a_{w}, w\right)$ rises in time for wealth smaller than but close to

Consider relative consumption $\chi(a) \equiv x(a) / y(a)$. By Lemma A.3, the number of sign changes of $\chi^{\prime}(a)$ in any interval for $a$ of finite length is finite. We can therefore for any $a_{0}$ find an $\varepsilon>0$ such that $\chi(a)$ is monotonic in $\left[a_{0}-\varepsilon, a_{0}\right]$. Exploiting this for $a_{w}^{*}$, whatever the 
properties of relative consumption, we can always find an $\varepsilon$ such that one of the following three cases must hold for $\Omega_{\varepsilon} \equiv\left[a_{w}^{*}-\varepsilon, a_{w}^{*}[\right.$,

$\left.\begin{array}{l}\text { (i) } \\ \text { (ii) }\end{array}\right\}\left.\chi^{\prime}(a)\right|_{a \in \Omega_{\varepsilon}}\left\{\begin{array}{l}< \\ > \\ =\end{array}\right\} 0$.

Note that we do not make any statement about the derivative in $a_{w}^{*}$. In fact, in case (i) $\left.\chi^{\prime}(a)\right|_{a \in a_{w}^{*}}$ can be negative or zero, in case (ii), it can be positive or zero.

Lemma A.5 (a) Consumption of employed workers rises over time for a wealth level a $\in \Omega_{\varepsilon}$ if and only if case (i) holds,

$$
\frac{d c\left(a_{w}(\tau), w\right)}{d \tau}>0 \text { for } a_{w}(\tau) \in \Omega_{\varepsilon} \Leftrightarrow \text { case (i) holds. }
$$

(b) Consumption $c\left(a_{w}(\tau), w\right)$ falls over time for $a_{w}(\tau) \in \Omega_{\varepsilon}$ if and only if (ii) holds.

Proof. (a) By (A.2), $\frac{d c\left(a_{w}(\tau), w\right)}{d \tau}>0 \Leftrightarrow c\left(a_{w}(\tau), w\right) / c\left(a_{w}(\tau), b\right)>1 / \psi$. As $c\left(a_{w}^{*}, w\right) / c\left(a_{w}^{*}, b\right)=$ $1 / \psi$ at $a_{w}^{*}$, as $w$ and $b$ are parameters and using ass. A.3, this is a condition on the derivative of relative consumption with respect to wealth $a$ in $\Omega_{\varepsilon}: d c\left(a_{w}(\tau), w\right) / d \tau$ is positive for $a_{w}(\tau) \in \Omega_{\varepsilon}$ if and only if case (i) holds.

(b) By (A.2), consumption falls over time if relative consumption lies below $1 / \psi$. This can be the case in $\Omega_{\varepsilon}$ only if case (ii) holds.

Lemma A.6 Relative consumption falls in wealth for $a \in \Omega_{\varepsilon},\left.\chi^{\prime}(a)\right|_{a \in \Omega_{\varepsilon}}<0$, i.e. case (i) holds.

Proof. a) Assume that case (ii) holds, i.e. $\left.\quad \chi^{\prime}(a)\right|_{a \in \Omega_{\varepsilon}}>0$. Then, by Lemma A.5, $\frac{d c\left(a_{w}(\tau), w\right)}{d \tau}<0$ for $a_{w}(\tau)<a_{w}^{*}$. Consumption of unemployed workers would still decrease in time for all wealth levels. In our set $Q_{v}$ from (12), $\frac{d a_{w}(\tau)}{d \tau}>0$ and therefore $\frac{d x(a)}{d a}<0$. As $\frac{d c\left(a_{b}(\tau), b\right)}{d \tau}<0$ and $\frac{d a_{b}(\tau)}{d \tau}<0$ in $Q_{v}$, we know that $\frac{d y(a)}{d a}>0$. As a consequence, $\chi^{\prime}(a)<0$. This contradicts the assumption that case (ii) holds and case (ii) can be excluded.

b) Now assume that case (iii) holds, i.e. relative consumption is flat, $\left.\chi^{\prime}(a)\right|_{a \in \Omega_{\varepsilon} \cup a_{w}^{*}}=0$. As $c\left(a_{w}^{*}, w\right) / c\left(a_{w}^{*}, b\right)=1 / \psi, d c\left(a_{w}(\tau), w\right) / d \tau=0$ for $a_{w}(\tau) \in \Omega_{\varepsilon}$. As $d c\left(a_{b}(\tau), b\right) / d \tau<0$, relative consumption is not constant - which contradicts the assumption that relative consumption is flat in wealth. As case (iii) is thereby excluded as well, the proof is complete.

- A global result

We now complete the proof by a global result on consumption growth.

Lemma A.7 Consumption $c\left(a_{w}, w\right)$ (a) rises in time for all $a<a_{w}^{*}$ and (b) decreases in time for all $a>a_{w}^{*}$.

Proof. (a) Imagine to the contrary of " $c\left(a_{w}, w\right)$ rises in time for all $a<a_{w}^{*}$ " that there is an interval $] \Gamma_{1}, \Gamma_{2}\left[\right.$ with $\Gamma_{2}<a_{w}^{*}$ such that this is is the last interval before $a_{w}^{*}$ where $c\left(a_{w}, w\right)$ falls in time,

$$
d c\left(a_{w}(\tau), w\right) / d \tau<0, \quad \forall \Gamma_{1}<a_{w}(\tau)<\Gamma_{2}<a_{w}^{*} .
$$

We now proceed as in the proof of Lemma A.6. As $\frac{d a_{w}(\tau)}{d \tau}>0$ in $Q_{v}$, this would imply that $\frac{d x(a)}{d a}<0$ for $\Gamma_{1}<a<\Gamma_{2}$. We know that $\frac{d y(a)}{d a}>0$ in $Q_{v}$. Hence, we would conclude that

$$
\chi^{\prime}(a)<0, \quad \forall \Gamma_{1}<a<\Gamma_{2} .
$$


By (A.2), the assumption in (A.7) would hold if and only if relative consumption $\frac{c\left(a_{w}, w\right)}{c\left(a_{w}, b\right)}$ is below $1 / \psi$ for $\Gamma_{1}<a<\Gamma_{2}$ : $\frac{d c\left(a_{w}(\tau), w\right)}{d \tau}<0 \Leftrightarrow \frac{c\left(a_{w}(\tau), w\right)}{c\left(a_{w}(\tau), b\right)}<1 / \psi$. As $\frac{x(a)}{y(a)}$ is continuous in wealth by Lemma A.2 and as case (i) holds by Lemma A.6, $\frac{x(a)}{y(a)}$ can be smaller than $1 / \psi$ only if there is some range $] \Gamma_{3}, \Gamma_{2}$ [ in which $\chi^{\prime}(a)>0$. (An example of such a path is shown in Figure 2.) This is a contradiction to the conclusion in (A.8). Hence, consumption must rise in time for all $a<a_{w}^{*}$.

(b) This proof is in analogy to the proof of (a).

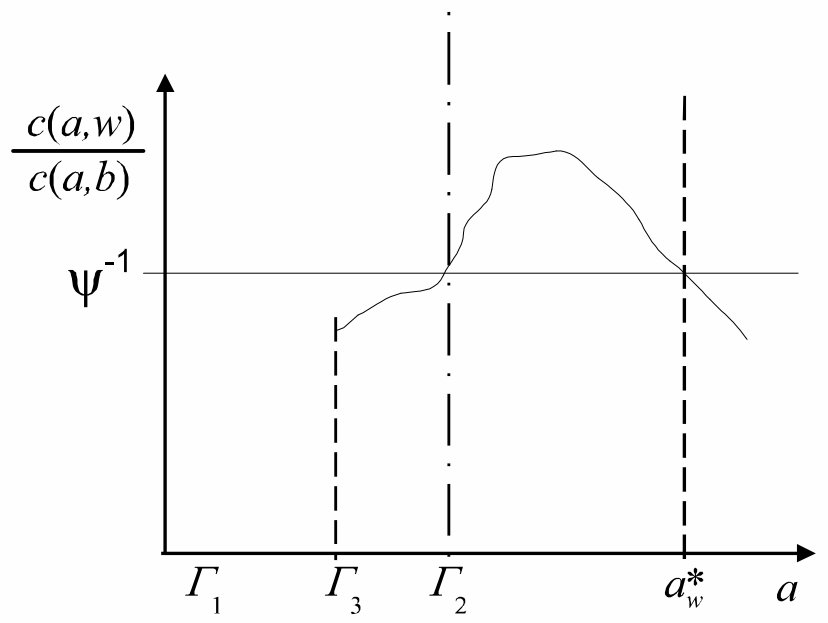

Figure 2 An example for relative consumption $\chi(a) \equiv \frac{x(a)}{y(a)}$

\section{A.3.2 Intermediate steps}

Before we prove the rest of Proposition A.4, we need some further intermediate results - which, however, are of some interest in their own right. Given that marginal utility from (1) is positive and decreasing, $u^{\prime}(c)>0$ and $u^{\prime \prime}(c)<0$, we can establish that $x(a)>y(a)$, i.e. consumption in the state of employment is larger than in the state of unemployment, keeping wealth constant. We prove in passing that the value functions $V(a, z)$ are strictly concave in wealth $a$.

Lemma A.8 Consumption rises in wealth, $c_{a}(a, z)>0$.

Proof. Proposition A.4 (i) shows that $d c\left(a_{w}(\tau), w\right) / d \tau>0$ in $Q_{v}$. As $d a_{w}(\tau) / d \tau>0$ as well, the derivative $d x(a) / d a$ in $(7)$ is positive in $Q_{v}$.

Lemma A.9 As marginal utility from consumption is positive, the value function $V(a, z)$ rises in wealth, $V_{a}(a, z)>0$.

Proof. The first-order condition for optimal consumption is given by (6) in the Referees' appendix and reads

$$
u^{\prime}(c(a, z))=V_{a}(a, z) .
$$

As marginal utility is positive by (1), the value function rises in wealth.

Lemma A.10 As $u^{\prime \prime}(c)<0$ and as consumption rises in a by Lemma A.8, the value function is strictly concave in a. 
Proof. The partial derivative of the first-order condition with respect to wealth implies

$$
u^{\prime \prime}(c(a, z)) c_{a}(a, z)=V_{a a}(a, z)
$$

As $u^{\prime \prime}(c(a, z))<0$ from the concavity of $(1)$ and $c_{a}(a, z)$ is positive by Lemma A.8, $V_{a a}(a, z)$ must be negative. With Lemma A.9, the value function is strictly concave.

Lemma A.11 The shadow price for wealth is higher in the state of unemployment, $V_{a}(a, b)>$ $V_{a}(a, w)$.

Proof. The derivation of the Keynes-Ramsey rule gives us (see Appendix B.1)

$$
\begin{aligned}
& (\rho-r) V_{a}(a, z)-s(z)\left[V_{a}(a, b)-V_{a}(a, w)\right]-\lambda(z)\left[V_{a}(a, w)-V_{a}(a, b)\right] \\
& =[r a+z-c(a, z)] V_{a a}(a, z) .
\end{aligned}
$$

In state $z=w$, this means

$$
(\rho-r) V_{a}(a, w)-s(z)\left[V_{a}(a, b)-V_{a}(a, w)\right]=[r a+w-x(a)] V_{a a}(a, w) .
$$

Given the region we are interested in (where $r a+w-x(a)>0$ ) and given Lemma A.10, the righthand side is negative. Hence, the left-hand side must be negative as well. As $(\rho-r) V_{a}(a, w)$ is positive due to $r<\rho$, the second term must be negative. This is the case only for $V_{a}(a, b)>$ $V_{a}(a, w)$.

Lemma A.12 Consumption of the employed worker is higher than consumption of the unemployed worker, $x(a)>y(a)$.

Proof. As $V_{a}(a, b)>V_{a}(a, w)$, the first-order condition implies $u^{\prime}(y(a))>u^{\prime}(x(a))$. As the marginal utility is decreasing, $x(a)>y(a)$.

\section{A.3.3 Proof of parts (ii) and (iii)}

(ii) By (A.4), $d c\left(a_{b}(\tau), b\right) / d \tau<0 \Leftrightarrow u^{\prime}\left(c\left(a_{b}(\tau), w\right)\right)<\varkappa u^{\prime}\left(c\left(a_{b}(\tau), b\right)\right)$ where $\varkappa \equiv 1-\frac{r-\rho}{\lambda} \geq 1$ as $r \leq \rho$. As $u^{\prime}\left(c\left(a_{b}(\tau), w\right)\right)<u^{\prime}\left(c\left(a_{b}(\tau), b\right)\right)$ with $c\left(a_{b}(\tau), w\right)>c\left(a_{b}(\tau), b\right)$ from Lemma A.12, this condition always holds.

(iii) This follows from solving (A.5) for relative consumption.

\section{A.4 Proof of Lemma 4.2 (existence of an optimal consumption path for $v>0$ )}

For simple reference in what follows and to simplify notation, define

$$
x(a) \equiv c(a, w), \quad y(a) \equiv c(a, b),
$$

and express the reduced form (7) as

$$
\begin{aligned}
& \dot{x}(a)=\frac{r-\rho+s\left[\left(\frac{x(a)}{y(a)}\right)^{\sigma}-1\right]}{r a+w-x(a)} \frac{x(a)}{\sigma}, \\
& \dot{y}(a)=\frac{r-\rho-\lambda\left[1-\left(\frac{y(a)}{x(a)}\right)^{\sigma}\right]}{r a+b-y(a)} \frac{y(a)}{\sigma} .
\end{aligned}
$$


The implication of the natural borrowing limit in (4) can be expressed here as

$$
y(-b / r)=0
$$

Any solution to (A.13) must satisfy this condition.

We study a regularized version of this ODE system in the sense that we restrict attention to the set $Q_{v}$ from (12). In addition we restrict the region further to make it bounded in order to avoid technical difficulties associated to the function $c(a, w)$ becoming arbitrarily large. We consider

$$
R_{v, \Psi}=\left\{(a, c(a, w), c(a, b)) \in \mathbb{R}^{3} \mid(a, c(a, w), c(a, b)) \in Q_{v}, c(a, w) \leq \Psi<\infty\right\},
$$

where $\Psi$ is a fixed positive constant which is sufficiently large. ${ }^{20}$ A third parameter $\varepsilon$ will also be introduced shortly to avoid the singularity at (A.14). This temporarily restricts our analysis further to the set

$$
R_{\varepsilon, v, \Psi}=R_{v, \Psi} \cap\left\{(a, x, y) \in \mathbb{R}^{3} \mid y \geq \varepsilon\right\}
$$

\section{A.4.1 Preliminaries}

In what follows, we will use classical theorems for initial value problems for ODEs. Currently, we have formulated our system (A.13) as a terminal value problem, since the definition of the optimal consumption path in Definition 4.1 uses a terminal condition $\left(a_{w}^{*}, c\left(a_{w}^{*}, w\right), c\left(a_{w}^{*}, b\right)\right)$ at the end of the interval $\left[-b / r, a_{w}^{*}\right]$ under consideration. Using the notation from (A.12) and given our focus on $R_{v, \Psi}$ from (A.15) in this section, this terminal condition can be written in compact form as

$$
\Phi \equiv \Phi_{v}(\hat{a})=\left(\hat{a}, x_{v}(\hat{a}), y_{v}(\hat{a})\right) .
$$

Note that $\Phi$ depends on $v$, i.e. $x_{v}(\hat{a})$ is defined in analogy to (11) as $x_{v}(\hat{a})=r \hat{a}+w-v$ and $y_{v}(\hat{a})$ is determined by $\left(x_{v}(\hat{a}) / y_{v}(\hat{a})\right)^{\sigma}=1-\frac{r-\rho}{s}$ in analogy to (8).

For ease of notation and to help intuition, we shall now recast the problem into a classical initial value problem, i.e. we will require the value $\Phi$ to be attained at the fixed beginning $\tau=0$ of an interval $\left[0, \tau^{*}\right]$, on which we study the problem. To this end, it is more useful to work with an autonomous system. Hence, we rewrite (A.13) by including $m(a)=a$ as third variable which "replaces" wealth $a$, which now purely serves as a parameter, i.e. as the independent variable. By using (A.12), this gives the system

$$
\begin{aligned}
& \dot{m}(a)=1 \\
& \dot{x}(a)=\frac{r-\rho+s\left[\left(\frac{x(a)}{y(a)}\right)^{\sigma}-1\right]}{r m(a)+w-x(a)} \frac{x(a)}{\sigma}, \\
& \dot{y}(a)=\frac{r-\rho-\lambda\left[1-\left(\frac{y(a)}{x(a)}\right)^{\sigma}\right]}{r m(a)+b-y(a)} \frac{y(a)}{\sigma} .
\end{aligned}
$$

Now define $\tau \equiv \hat{a}-a, x_{1}(\tau) \equiv m(\hat{a}-\tau), x_{2}(\tau) \equiv x(\hat{a}-\tau), x_{3}(\tau) \equiv y(\hat{a}-\tau)$. Then, $\frac{d}{d \tau} x_{1}(\tau) \equiv \dot{x}_{1}(\tau)=\frac{d}{d \tau} m(\hat{a}-\tau)=\frac{d}{d[\hat{a}-a]} m(a)=-\frac{d}{d a} m(a)=-\dot{m}(a)$. Doing the same for $x$

\footnotetext{
${ }^{20}$ The constant $\Psi$ only serves to make $R_{v} \Psi \subset \mathbb{R}^{3}$ a compact set, which we need to obtain global, uniform Lipschitz constants. We shall see below that $\Psi$ has to be chosen larger than $\Psi_{0}=\frac{\psi w-b}{(1-\psi) r}$. In this case, however, $\Psi$ does not interfere with the construction.
} 
and $y$, the "inverted" autonomous system therefore reads

$$
\begin{aligned}
& \dot{x}_{1}(\tau)=-1 \\
& \dot{x}_{2}(\tau)=-\frac{r-\rho+s\left[\left(\frac{x_{2}(\tau)}{x_{3}(\tau)}\right)^{\sigma}-1\right]}{r x_{1}(\tau)+w-x_{2}(\tau)} \frac{x_{2}(\tau)}{\sigma}, \\
& \dot{x}_{3}(\tau)=-\frac{r-\rho-\lambda\left[1-\left(\frac{x_{3}(\tau)}{x_{2}(\tau)}\right)^{\sigma}\right]}{r x_{1}(\tau)+b-x_{3}(\tau)} \frac{x_{3}(a)}{\sigma},
\end{aligned}
$$

where now $\dot{x}_{i}$ denotes the derivative of $x_{i}(\tau)$ with respect to $\tau, i=1,2,3$.

Definition A.13 Given (A.18) and for $\tau \geq 0$, let $X(\tau ; \Phi)=\left(x_{1}(\tau), x_{2}(\tau), x_{3}(\tau)\right)$ denote the solution of (A.18) started at $X(0 ; \Phi)=\Phi \in R_{v, \Psi}$ from (A.17) where $-b / r \leq \hat{a} \leq \frac{\Psi+v-w}{r}$. For later use, we also introduce the notation $x_{i}(\tau)=x_{i}(\tau ; \Phi), i=1,2,3$.

By passing from (A.13) to (A.18) we have reversed the time-direction - more precisely, in our setting, the wealth-direction - and turned a non-autonomous system into an autonomous one by including the independent variable as an additional component of the solution. Thus, the curve $a \mapsto(a, x(a), y(a))$ with terminal value $x(\hat{a})=x_{v}(\hat{a}), y(\hat{a})=y_{v}(\hat{a})$ is equal to the curve $\tau \mapsto X(\tau ; \Phi)$ with $\Phi=\Phi(\hat{a})$, which is the solution of an initial value problem in the classical sense. However, the parametrization is reverted in the sense that in the former case we start at the left endpoint ("left" in the sense of the smallest value of the $a$-component) and end in the right endpoint, whereas in the latter case we start at the right endpoint and end in the left one. In particular, the absolute value of the speed along the curve is equal, but the direction is reversed.

\section{A.4.2 Continuity of the solution in initial values}

In order to be able to apply classical theorems, we need finite derivatives on the right-hand side of an ODE system. The right-hand side of the ODE (A.13), however, exhibits singularities at the boundary $y=r a+b$ of $Q_{v}$. This is of particular importance as the definition of the optimal consumption path in Definition 4.1 uses $y(-b / r)=0$ - which lies on this boundary. We obtain finite derivatives by (i) a coordinate transformation and by (ii) (temporarily) reducing the set on which we are interested in a solution by demanding that $y \geq \varepsilon$. We will later show (see remark A.23) how this reduction can then be removed again by passing $\varepsilon \rightarrow 0$.

Lemma A.14 (Coordinate transformation) Let $x(a)$ and $y(a)$ be solutions of (A.13). The mapping $a \mapsto y(a)$ is bijective. Change variables $a=a(y)$ and consider $x$ and $a$ as functions of $y$. Then

$$
\begin{aligned}
& x^{\prime}(y) \equiv \frac{d x(y)}{d y}=\frac{r-\rho+s\left[\left(\frac{x(y)}{y}\right)^{\sigma}-1\right]}{r-\rho-\lambda\left[1-\left(\frac{y}{x(y)}\right)^{\sigma}\right]} \frac{x(y)}{y} \frac{r a(y)+b-y}{r a(y)+w-x(y)}, \\
& a^{\prime}(y) \equiv \frac{d a(y)}{d y}=\frac{r a(y)+b-y}{r-\rho-\lambda\left[1-\left(\frac{y}{x(y)}\right)^{\sigma}\right]} \frac{\sigma}{y} .
\end{aligned}
$$

Proof. Since $\dot{y}(a)>0, y$ is a bijective function of $a$. As $a^{\prime}(y)=\frac{1}{\dot{y}(a)}$, we obtain the second equation by inserting (A.13b). The first equation follows from "dividing (A.13a) by (A.13b)".

We are going to avoid the singularity at $y(-b / r)=0$ by temporarily requiring (see remark A.23 below) these properties to hold only "up to an arbitrarily small number $\varepsilon$ ". We do this by considering the domain $R_{\varepsilon, v, \Psi}$ as given in the following 
Definition A.15 Fix a numbers $\varepsilon>0$ and define $R_{\varepsilon, v, \Psi}$ from (A.16), reproduced here for convenience,

$$
R_{\varepsilon, v, \Psi}=R_{v, \Psi} \cap\left\{(a, x, y) \in \mathbb{R}^{3} \mid y \geq \varepsilon\right\} .
$$

This definition implies that we temporarily replace the requirement that $y(-b / r)=0$ by $y(a)=\varepsilon$ for some $-b / r \leq a \leq-b / r+\varepsilon / r$.

Lemma A.16 The right-hand side given in (A.19) is uniformly Lipschitz on $R_{\varepsilon, v, \Psi}$.

Proof. Consider the right-hand side of (A.19a). The only possible points, where the Lipschitz constant can explode, are when the denominators in the right-hand side become 0 or when a term under a fractional power (i.e. with exponent $\sigma$ ) becomes 0 . In $R=R_{\varepsilon, v, \Psi}, y$ is uniformly bounded away from 0 and $x$ is uniformly bounded away from $r a+w$. Moreover, note that $r-\rho-\lambda\left[1-\left(\frac{y}{x}\right)^{\sigma}\right]=0$ if and only if $\left(\frac{y}{x}\right)^{\sigma}=1-\frac{r-\rho}{\lambda}$. Now $1-\frac{r-\rho}{\lambda}>1$ by the assumption that $r<\rho$. On the other hand, $y<x$, implying that $\left(\frac{y}{x}\right)^{\sigma}<1$. Consequently, all the denominators are uniformly bounded away from 0 .

For the fractional powers, note that $x / y>1$ is trivially uniformly bounded away from 0 . As $x \leq \Psi$,

$$
\frac{y}{x}>\frac{\epsilon}{\Psi}
$$

is uniformly bounded away from 0 on $R_{\varepsilon, v, \Psi}$. This shows that (A.19a) is uniformly Lipschitz.

The same arguments show that the right-hand side of (A.19b) is uniformly Lipschitz, too.

Since the right hand side of (A.19) is uniformly Lipschitz, we can now apply the classical theory of ODEs. For instance, we have existence and uniqueness of the solution by the PicardLindelöf theorem, see Mattheij and Molenaar (2002, Theorem II.2.3, Theorem II.3.1). Moreover, the solution will be continuous as a function of the initial value, see, again, Mattheij and Molenaar (2002, Theorem II.4.7). In the lemma below, we will see how this even implies the corresponding properties for the non-transformed system (A.18).

Lemma A.17 (Continuity in initial values) Consider the set $R=R_{\varepsilon, v, \Psi}$ from (A.16) and the solution $X(\tau ; \Phi)$ from Definition A.13 with initial condition $\Phi$ given in (A.17). The solution $X(\tau ; \Phi)$ depends continuously on its initial values $\Phi$. More precisely, there is a constant $L>0$ and an increasing map $\kappa:\left[0, \infty\left[\rightarrow\left[0, \infty\left[\right.\right.\right.\right.$ (a modulus of continuity) with $\lim _{t \backslash 0} \kappa(t)=\kappa(0)=0$ such that

$$
\left\|X\left(\tau_{1} ; \Phi_{1}\right)-X\left(\tau_{2} ; \Phi_{2}\right)\right\| \leq L\left\|\Phi_{1}-\Phi_{2}\right\|+\kappa\left(\left|\tau_{1}-\tau_{2}\right|\right),
$$

provided that $\Phi_{1}, \Phi_{2} \in R$ and $X\left(\tau ; \Phi_{i}\right) \in R$ for all $0 \leq \tau \leq \max \left(\tau_{1}, \tau_{2}\right), i=1,2$. Here, $\|\cdot\|$ denotes the Euclidean norm on $\mathbb{R}^{3}$.

Proof. By classical results from the theory of ordinary differential equations, see for instance Mattheij and Molenaar (2002, Theorem II.4.7), the solution of an ODE-system depends continuously on the initial data as long as the right-hand side is uniformly Lipschitz. More precisely, let $Y(\tau ; \Phi)$ denote the solution of an ODE with uniformly Lipschitz right-hand side (with Lipschitz constant $C$ ), started at $Y\left(\tau_{0} ; \Phi\right)=\Phi$, then

$$
\left\|Y\left(\tau ; \Phi_{1}\right)-Y\left(\tau ; \Phi_{2}\right)\right\| \leq \exp \left(C\left(\tau-\tau_{0}\right)\right)\left\|\Phi_{1}-\Phi_{2}\right\| .
$$

Now consider the transformed system $(a(y), x(y))$ from (A.19). By Lemma A.16, the righthand side is uniformly Lipschitz. The solution of (A.19) therefore depends continuously on its initial data $\left(a_{0}, x_{0}\right)$. It is then obvious that the trajectory $(a(y), x(y), y)$ depends continuously on $\left(a_{0}, x_{0}, y_{0}\right)$. As system (A.19) is a reparameterized version of (A.13), the solution 
$(a, x(a), y(a))$ to (A.13) from Definition 4.1 is also continuous in its boundary conditions even though the right hand side of (A.13) is not uniformly Lipschitz. Similarly, as (A.18) is just a reparameterization of (A.13), the solution $X(\tau ; \Phi)$ to (A.18) from Definition A.13 is also continuous in its initial condition $\Phi$.

In order to get the estimate, we now consider the ODE (A.18) and note that we only consider it on the compact set $R_{\varepsilon, v, \Psi}$. In the parametrization by $y$ given in (A.19), $y$ is the independent variable, i.e. plays the role of $\tau$ in the above estimate. By compactness of $R_{\varepsilon, v, \Psi}, y$ only runs through a bounded set, therefore we can rewrite the constant in the above inequality as $\exp \left(C\left(y-y_{0}\right)\right) \leq L$ for some suitable $L>0$.

Given $\Phi \in R_{\varepsilon, v, \Psi}$. Then $a_{w}^{*} \leq \frac{\Psi-w+v}{r}$, which implies that the solution $X(\tau ; w)$ can only stay inside $R_{\varepsilon, v, \Psi}$ until time $\tau=\frac{\Psi-w+v+b}{r}$, at most. Consider

$$
D=\left\{( \tau , \Phi ) \in \left[0, \infty\left[\times R_{\varepsilon, v, \Psi} \mid X(\tau ; \Phi) \in R_{\varepsilon, v, \Psi}\right\} .\right.\right.
$$

Then $D$ is a closed subset of $\left[0, \frac{\Psi-w+v+b}{r}\right] \times R_{\varepsilon, v, \Psi}$, implying that $D$ is compact. Consequently, $X: D \rightarrow R_{\varepsilon, v, \Psi}$ is uniformly continuous, which implies the existence of a modulus of continuity $\kappa$ with

$$
\left\|X\left(\tau_{1} ; \Phi_{1}\right)-X\left(\tau_{2} ; \Phi_{2}\right)\right\| \leq \kappa\left(\left|\tau_{1}-\tau_{2}\right|+\left\|\Phi_{1}-\Phi_{2}\right\|\right)
$$

The inequality in the lemma then follows by the triangle inequality.

\section{A.4.3 Continuity of the first hitting-wealth in initial values}

While we have shown in the previous section that the solutions to all systems (A.13), (A.18) and (A.19) are continuous in initial values, this does not automatically imply that the solutions will be continuous on the boundary of the domain we are interested in, in the sense that the place where the solution leaves the domain $R$ might not depend continuously on the initial data. This will now be proved in this section.

In the proofs and also in a later step, we will use the following

Definition A.18 (First hitting-wealth) Consider the set $R_{\varepsilon, v, \Psi}$ from (A.16) and the solution $X(\tau ; \Phi)$ to the system (A.18). Consider the path $y(a)$ that corresponds to $x_{2}(\tau)$ of this solution. Then we define $\hat{a}_{1 s t}=f(\hat{a})$ as the "first hitting-wealth" (in analogy to first hitting-time), i.e. the wealth level where the path $y(a)$ hits any boundary of $R_{\varepsilon, v, \Psi}$ for the first time. Similarly denote $\tau(\Phi) \equiv \inf \left\{\tau \geq 0 \mid X(\tau ; \Phi) \in \partial R_{\varepsilon, v, \Psi}\right\}$ and $F(\Phi) \equiv X(\tau(\Phi) ; \Phi)$.

We know that $\hat{a}_{1 s t}$ exists because in the set $R_{\varepsilon, v, \Psi}$ the derivatives in (A.18) are well-defined and a solution therefore exists. Notice that $\hat{a}_{1 s t}$ equals the first component of $F(\Phi(\hat{a}))$.

We also need

Definition A.19 Let $N \subset R_{\varepsilon, v, \Psi}$ with

$$
N=\left\{\Phi(\hat{a}) \mid \hat{a} \in\left[-\frac{b}{r}, \frac{\psi[w-v]-b}{r[1-\psi]}\right]\right\}
$$

be the set of all potential initial conditions from (A.17) for a solution in the sense of Definition 4.1. Here we implicitly assume that $\Psi$ is large enough that indeed $N \subset R_{\varepsilon, v, \Psi} \cdot{ }^{21}$ Define $M$ as

$$
M=M_{1} \cup M_{2} \cup M_{3} \subset R_{\varepsilon, v, \Psi}
$$

\footnotetext{
${ }^{21}$ This is the only necessary condition on $\Psi$ for the construction to work. In the sequel, we shall assume this condition without further notice.
} 
with

$$
\begin{aligned}
& M_{1}=\left\{(a, x, y) \in R_{\varepsilon, v, \Psi} \mid y=r a+b\right\}, \\
& M_{2}=\left\{(a, x, y) \in R_{\varepsilon, v, \Psi} \mid a=-b / r\right\}, \\
& M_{3}=\left\{(a, x, y) \in R_{\varepsilon, v, \Psi} \mid y=\varepsilon\right\} .
\end{aligned}
$$

This set will turn out to be the set of all potential first hitting-wealths.

Since we know that $x>y$, the trajectory will not hit the boundary of $R$ at the part $\{x=y\}$. Therefore, we have the

Corollary A.20 $F: N \rightarrow M$ is a well-defined map, i.e. for every $\Phi \in N$, the corresponding solution path $X(\tau ; \Phi)$ exists and stays in $R_{\varepsilon, v, \Psi}$ until it finally hits $M$ (and no other boundary of $\left.R_{\varepsilon, v, \Psi}\right)$.

Before formulating the main lemma of this section, let us first derive a simple bound on the derivative $\dot{y}(a)$ of the consumption of the unemployed.

Lemma A.21 For $(a, x, y)$ in the interior of $Q_{v}$ from (12), we have

$$
\dot{y}(a) \geq \frac{r-\rho}{r a+b-y(a)} \frac{y(a)}{\sigma} .
$$

Proof. By (A.13b) we have

$$
\begin{aligned}
\dot{y}(a)=\frac{r-\rho-\lambda\left[1-\left(\frac{y(a)}{x(a)}\right)^{\sigma}\right]}{r a+b-y(a)} \frac{y(a)}{\sigma} & \left(\frac{r-\rho}{r a+b-y(a)}-\frac{\lambda\left[1-\left(\frac{y(a)}{x(a)}\right)^{\sigma}\right]}{r a+b-y(a)}\right) \frac{y(a)}{\sigma}>\frac{r-\rho}{r a+b-y(a)} \frac{y(a)}{\sigma} .
\end{aligned}
$$

The last inequality follows from the fact that $\frac{\lambda\left[1-\left(\frac{y(a)}{x(a)}\right)^{\sigma}\right]}{r a+b-y(a)}$ is negative (and therefore $-\frac{\lambda\left[1-\left(\frac{y(a)}{x(a)}\right)^{\sigma}\right]}{r a+b-y(a)}$ is positive) as $r a+b-y(a)$ is negative in the interior of $Q_{v}$.

The key result in this section is presented in

Lemma A.22 The map $F: N \rightarrow M$ is continuous.

Proof. We need to prove that for every $\Phi \in N$ and every $\delta>0$ there is an $\eta>0$ such that

$$
\left\|\Phi_{0}-\Phi\right\|<\eta \Longrightarrow\left\|F\left(\Phi_{0}\right)-F(\Phi)\right\|<\delta .
$$

We start the proof by fixing $\Phi_{0}, \Phi \in N$ such that $\left\|\Phi_{0}-\Phi\right\|<\eta$ for some $\eta>0$. Let us first assume that $\tau\left(\Phi_{0}\right) \leq \tau(\Phi)$. By the triangle inequality and Lemma A.17, we have

$$
\begin{aligned}
\left\|X\left(\tau\left(\Phi_{0}\right) ; \Phi_{0}\right)-X(\tau(\Phi) ; \Phi)\right\| \leq & \left\|X\left(\tau\left(\Phi_{0}\right) ; \Phi_{0}\right)-X\left(\tau\left(\Phi_{0}\right) ; \Phi\right)\right\|+ \\
& +\left\|X\left(\tau\left(\Phi_{0}\right) ; \Phi\right)-X(\tau(\Phi) ; \Phi)\right\| \\
\leq & L_{1}\left\|\Phi_{0}-\Phi\right\|+\kappa\left(\left|\tau\left(\Phi_{0}\right)-\tau(\Phi)\right|\right)
\end{aligned}
$$

for a constant $L_{1}>0$ and the modulus of continuity $\kappa$. In order to get an estimate for $\left|\tau\left(\Phi_{0}\right)-\tau(\Phi)\right|$, we have to distinguish between three different cases.

Case (i): $F\left(\Phi_{0}\right) \in M_{1}$. 
By Lemma A.21, there are constants $L_{2}, \ell_{2}>0$ such that $\dot{y} \geq L_{2}$ for $|y-(r a+b)| \leq \ell_{2}$. More precisely, we can choose $\ell_{2}>0$ freely and obtain the bound for $L_{2}=\frac{1}{\ell_{2}} \frac{(\rho-r) \varepsilon}{\sigma}$. If $L_{1} \eta \leq \ell_{2}$, we can bound the absolute value of the derivative of $x_{3}(\tau ; \Phi)$ from below by $L_{2}$ (for $t \geq \tau\left(\Phi_{0}\right)$ ). This implies that the path $X(\tau ; \Phi)$ hits $M_{1}$ before time $\tau\left(\Phi_{0}\right)+\tau$ for

$$
\tau\left(L_{2}-r\right)=\ell_{2} \Longleftrightarrow \tau=\frac{\ell_{2}}{L_{2}-r},
$$

unless it hits another boundary of $R_{\varepsilon, v, \Psi}$ before that. Inserting into (A.22), this gives the estimate

$$
\left\|F\left(\Phi_{0}\right)-F(\Phi)\right\| \leq L_{1} \eta+\kappa\left(\frac{\ell_{2}}{L_{2}-r}\right) .
$$

Choosing $\ell_{2}=L_{1} \eta$, the bound is smaller than $\delta$ provided that

$$
\kappa\left(\frac{L_{1}}{\frac{C}{L_{1} \eta}-r} \eta\right)+L_{1} \eta<\delta,
$$

where $C \equiv \frac{(\rho-r) \varepsilon}{\sigma}$. Note that the left hand side in (A.23) converges to zero for $\eta \rightarrow 0$, therefore we can find an $\eta_{0}(\delta)>0$ (only depending on the constants $C, L_{1}$ and $r$ and the modulus of continuity $\kappa$, but not on $\Phi_{0}$ or $\Phi$ ) such that the desired inequality (A.21) holds for $\eta<\eta_{0}$. We have tacitly assumed that $L_{2}=C / \ell_{2}=\frac{C}{L_{1} \eta}>r$, which can be realized by choosing $\eta$ small enough.

Case (ii): $F\left(\Phi_{0}\right) \in M_{2}$.

Let $\hat{a}$ denote the first component of $\Phi$, and $\hat{a}_{0}$ the first component of $\Phi_{0}$. Note that $x_{1}(\tau ; \Phi)=$ $\hat{a}-\tau$, for every $\tau \geq 0$. Since $X\left(\tau\left(\Phi_{0}\right) ; \Phi_{0}\right) \in M_{2}$, we have $-b / r=x_{1}\left(\tau\left(\Phi_{0}\right) ; \Phi_{0}\right)=\hat{a}_{0}-\tau\left(\Phi_{0}\right)$, implying that $\tau\left(\Phi_{0}\right)=\hat{a}_{0}+b / r$. On the other hand, $x_{1}(\tau(\Phi) ; \Phi) \geq-b / r$, implying that $\tau(\Phi) \leq \hat{a}+b / r$. Combining these two results, we obtain

$$
\left|\tau\left(\Phi_{0}\right)-\tau(\Phi)\right|=\tau(\Phi)-\tau\left(\Phi_{0}\right) \leq \hat{a}-\hat{a}_{0} \leq\left\|\Phi_{0}-\Phi\right\| .
$$

Consequently, the inequality (A.22) implies

$$
\left\|F\left(\Phi_{0}\right)-F(\Phi)\right\| \leq L_{1}\left\|\Phi_{0}-\Phi\right\|+\kappa\left(\left\|\Phi_{0}-\Phi\right\|\right) \leq L_{1} \eta+\kappa(\eta),
$$

and (A.21) holds for $\eta$ small enough such that

$$
L_{1} \eta+\kappa(\eta)<\delta
$$

Case (iii): $F\left(\Phi_{0}\right) \in M_{3}$.

Since $x_{3}\left(\tau\left(\Phi_{0}\right) ; \Phi_{0}\right)=\varepsilon$, we have $0 \leq x_{3}\left(\tau\left(\Phi_{0}\right) ; \Phi\right)-\varepsilon \leq L_{1} \eta$. By Lemma A.21, we can find a constant $L_{3}>0$ such that $\dot{y} \geq L_{3}$ on $R_{\varepsilon, v, \Psi}$ - note that $L_{3}$ depends on $\varepsilon$. Thus, $X(s ; \Phi)$ will hit the boundary $M_{3}$ before time $\tau\left(\Phi_{0}\right)+\tau$ with $\tau=L_{1} \eta / L_{3}$, unless it hits another boundary of $R_{\varepsilon, v, \Psi}$ before. In any case, $\left|\tau\left(\Phi_{0}\right)-\tau(\Phi)\right| \leq L_{1} \eta / L_{3}$, and we obtain

$$
\left\|F\left(\Phi_{0}\right)-F(\Phi)\right\| \leq L_{1} \eta+\kappa\left(\frac{L_{1}}{L_{3}} \eta\right)
$$

and (A.21) is satisfied for

$$
L_{1} \eta+\kappa\left(\frac{L_{1}}{L_{3}} \eta\right)<\delta
$$

Choosing $\eta$ small enough that both (A.23) and (A.24) and (A.25) are satisfied, settles the proof for $\tau\left(\Phi_{0}\right) \leq \tau(\Phi)$. Notice that none of the conditions (A.23), (A.24) and (A.25) depends on $\Phi_{0}$. Therefore, in the other case $\tau\left(\Phi_{0}\right) \geq \tau(\Phi)$, we can just revert the rôles of $\Phi$ and $\Phi_{0}$ and obtain the same results in cases (i), (ii) and (iii). 


\section{A.4.4 Existence of a solution for $v>0$}

This section proves our main result formulated in Theorem 4.3 regularized for $v>0$, i.e. for the set $R_{v, \Psi}$ from (A.15) and the terminal condition from (A.17).

Proof. Fix some $\varepsilon>0$ and consider $R_{\varepsilon, v, \Psi}$. By an intermediate value theorem applied to $F: N \rightarrow M$, we will obtain a point or points $\Phi \in N$ such that $F(\Phi) \in M_{3}$ as used in (A.20), i.e. $x_{3}(\tau(\Phi) ; \Phi)=\varepsilon$ provided that we can show the existence of points (that could be called upper and lower bounds) $\Phi_{v}^{\min }, \Phi_{v}^{\max } \in N$ with $F\left(\Phi_{v}^{\min }\right) \in M_{2}$ and $F\left(\Phi_{v}^{\max }\right) \in M_{1}$. (Note that $F=F_{\varepsilon}$ and all the $M_{i}=M_{i}(\varepsilon), i=1,2,3$, depend on $\varepsilon$ and $v$, but not on $\Psi$, provided that $\Psi$ is large enough.)

Choose

$$
\Phi_{v}^{\min }=\Phi(-b / r)=(-b / r, w-b-v, \psi[w-b-v]), \quad \Phi_{v}^{\max }=\Phi\left(\frac{\psi(w-v)-b}{(1-\psi) r}\right) .
$$

By construction, both $\Phi_{v}^{\min }$ and $\Phi_{v}^{\max }$ are contained in $N$. Moreover, we trivially have $F_{\varepsilon}\left(\Phi_{v}^{\min }\right) \in$ $M_{2}(\varepsilon), F_{\varepsilon}\left(\Phi_{v}^{\max }\right) \in M_{1}(\varepsilon)$ for every $\varepsilon>0$ small enough. Note, in particular, that Lemma A.22 also implies continuity of $F$ in the boundary points $\Phi_{v}^{\min }$ and $\Phi_{v}^{\max }$ of $N$. Therefore, the image set $F_{\varepsilon}(N)$ is a connected set, with non-empty intersection with both $M_{1}$ and $M_{2}$. Since the distance

$$
\operatorname{dist}\left(M_{1}, M_{2}\right)=\inf \left\{\left\|\Phi_{1}-\Phi_{2}\right\| \mid \Phi_{1} \in M_{1}, \Phi_{2} \in M_{2}\right\}=\frac{\varepsilon}{r}>0,
$$

we may conclude that $F_{\varepsilon}(N) \cap M_{3}(\varepsilon) \neq \emptyset$. This establishes that there must be a $\Phi$ such that $F_{\varepsilon}(\Phi) \in M_{3}$. In words, there is an initial condition $\Phi(\hat{a})$ such that the path $(a, x(a), y(a))$ hits the boundary at $y=\varepsilon$.

Now define

$$
N_{3}(\varepsilon) \equiv F_{\varepsilon}^{-1}\left(M_{3}(\varepsilon)\right)=\left\{\Phi \in N \mid F_{\varepsilon}(\Phi) \in M_{3}(\varepsilon)\right\} .
$$

By continuity of $F_{\varepsilon}: N \rightarrow M(\varepsilon)$, the bounded set $N_{3}(\varepsilon)$ is closed and thus compact. Moreover, the family $\left(N_{3}(\varepsilon)\right)_{\varepsilon>0}$ is directed in the sense that

$$
0<\varepsilon_{2}<\varepsilon_{1} \Longrightarrow N_{3}\left(\varepsilon_{2}\right) \subset N_{3}\left(\varepsilon_{1}\right) \text {. }
$$

By standard results from topology, the intersection of a directed family of non-empty, compact sets is non-empty, i.e.

$$
N_{3}(0) \equiv \bigcap_{\varepsilon>0} N_{3}(\varepsilon) \neq \emptyset .
$$

Indeed, take a decreasing sequence $\left(\varepsilon_{n}\right)_{n \geq 1}$ of positive numbers converging to zero. For every $n$ choose some $\Phi_{n} \in N_{3}\left(\varepsilon_{n}\right)$. By compactness of the largest set $N_{3}\left(\varepsilon_{1}\right)$, we can find a subsequence $n_{k}$ such that $\left(\Phi_{n_{k}}\right)_{k \geq 1}$ converges to some $\Phi$. Note that $\Phi \in N_{3}\left(\varepsilon_{n_{k}}\right)$ for every $k$, since $\Phi=$ $\lim _{l \rightarrow \infty, l \geq k} \Phi_{n_{l}}$ and each such $\Phi_{n_{l}}$ lies in the closed set $N_{3}\left(\varepsilon_{n_{k}}\right)$. Now choose any $\varepsilon>0$ and pick a $k$ such that $\varepsilon_{n_{k}}<\varepsilon$. Then $\Phi \in N_{3}\left(\varepsilon_{n_{k}}\right) \subset N_{3}(\varepsilon)$, implying that $\Phi \in \bigcap_{\varepsilon>0} N_{3}(\varepsilon)$.

We claim that every element $\Phi \in N_{3}(0)$ satisfies the requirements of an optimal consumption path regularized by $v>0$. Indeed, the path $(a, x(a), y(a))$ with terminal value $(\hat{a}, \hat{x}, \hat{y})=\Phi$ (corresponding to the path $X(\tau ; \Phi))$ satisfies the ODE (A.13) on $]-b / r, \hat{a}]$. Moreover, it starts at $N$ by construction, and for every $\varepsilon>0$, it takes on the value $\varepsilon$ somewhere on the interval ]$-b / r,-b / r+\varepsilon[$. Thus, using monotonicity of $y$, we may conclude that

$$
\lim _{a \searrow-b / r} y(a)=0 \text {. }
$$

This establishes that there is an initial condition $\Phi(\hat{a})$ such that the path $y(a)$ hits the boundary at $y=0$ in the sense that $y(-b / r)=0$. 
Remark A.23 Note that it is essential for the proof of Theorem 4.3 that the trajectory $X(\tau ; \Phi)$ - or, equivalently, $(a, x(a), y(a))$ - does not depend on $\varepsilon$, which only determines "how long" we observe the trajectory. This means that we observe the trajectory $X(\tau ; \Phi)$ for $0 \leq \tau \leq \tau(\Phi)$, with the hitting time $\tau(\Phi)$ obviously depending on $\varepsilon$. Therefore, we can, for fixed $\Phi \in N_{3}(0)$, easily take the limit $\varepsilon \rightarrow 0$, which means that we take the limit in $\tau(\Phi)$, but do not change the trajectory itself. As a consequence, the ODE is automatically satisfied for the limit, at least for $0 \leq \tau<\lim _{\varepsilon \rightarrow 0} \tau(\Phi)$.

Let us illustrate why we had to use the specific properties of the dynamic system (A.18) in the proof of Lemma A.22. Continuity in initial conditions does not imply continuity of "first hitting values" in general. Indeed, the first hitting times are inherently non-continuous functionals, even if both the paths and the set, which determines the hitting times, are smooth.

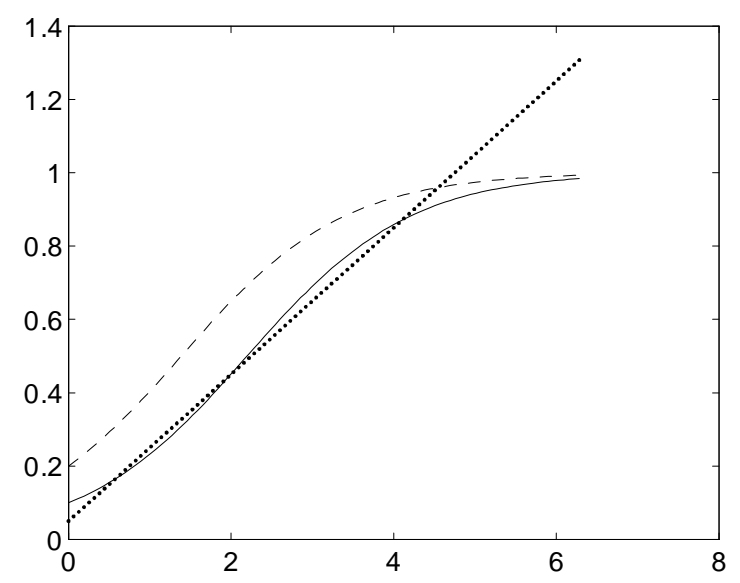

Figure 3 Non-continuity of the first hitting time

To see this most clearly, consider the differential equation $\dot{z}(t)=(1-z(t)) z(t)$ whose solution is $z(t)=\left(1+\left(z_{0}^{-1}-1\right) e^{-t}\right)^{-1}$. This solution is continuous in the initial level $z_{0}$ (for $z_{0}>0$ which we assume) and the solution is plotted for $z_{0} \in\{0.1,0.2\}$ in Figure 3. Now consider the first-hitting time on the straight line $0.05+t / 5$ as drawn. Obviously, this time is not continuous in the initial values $z_{0}$.

\section{A.5 Proof of Theorem 4.3 (existence of an optimal consumption path for $v=0$ )}

The previous section proved an existence theorem for the regularized problem with $v>0$. In this section it is shown that there is a sequence $v_{n}$ of values of $v$ with $v_{n} \rightarrow 0$ for $n \rightarrow \infty$ such that the solutions for the regularized problem converge to a solution of the original problem for the existence an optimal consumption path as $n \rightarrow \infty$. In the case that the existence of a solution on the interval $\left[-b / r, a_{w}^{*}\right]$ is known we can express the solution in terms of the reversed independent variable $\tau=a_{w}^{*}-a$ as before. The transformed solution then exists on the interval $\left[0, a_{w}^{*}+b / r\right]$. Note that the quantity $a_{w}^{*}$ may depend on the parameter $v$ and thus we sometimes denote it by $a_{w, v}^{*}$ or abbreviate $a_{w, v_{n}}^{*}$ by $a_{n}^{*}$. We now consider the equations written in terms of the variable $\tau$ and look for solutions of those equations on the interval $\left[0, a_{w}^{*}+b / r\right]$. Since we are looking for solutions satisfying particular boundary conditions for $a \rightarrow a_{w}^{*}$, we introduce new functions $X$ and $Y$ by the relations

$$
\begin{aligned}
& \tau X(\tau)=x\left(a_{w}^{*}-\tau\right)-r a_{w}^{*}-w+v \\
& \tau Y(\tau)=y\left(a_{w}^{*}-\tau\right)-\psi\left(r a_{w}^{*}+w-v\right)
\end{aligned}
$$


If $x$ and $y$ are functions defined on $\left(-b / r, a_{w}^{*}\right)$ which have smooth extensions to $a=a_{w}^{*}$ and satisfy the desired boundary conditions, then $X$ and $Y$ are defined on $\left(0, a_{w}^{*}+b / r\right)$ and bounded on the intervals of the form $(0, a)$ with $a>0$. Thus the aim is to find suitably bounded solutions of the equations obtained by rewriting the equations for $x(a)$ and $y(a)$ in terms of $X(\tau)$ and $Y(\tau)$. This gives

$$
\begin{aligned}
\tau \frac{d X}{d \tau}+X & =-\frac{r-\rho+s\left[\left(\frac{r a_{w}^{*}+w-v+\tau X(\tau)}{\psi\left(r a_{w}^{*}+w-v\right)+\tau Y(\tau)}\right)^{\sigma}-1\right]}{-r \tau+w-\tau X(\tau)} \frac{r a_{w}^{*}+w-v+\tau X(\tau)}{\sigma}, \\
\tau \frac{d Y}{d \tau}+Y & =-\frac{r-\rho-\lambda\left[1-\left(\frac{\psi\left(r a_{w}^{*}+w-v\right)+\tau Y(\tau)}{r a_{w}^{*}+w-v+\tau X(\tau)}\right)^{\sigma}\right]}{r\left(a_{w}^{*}-\tau\right)+b+\psi\left(\tau a_{w}^{*}+w-v\right)-\tau Y(\tau)} \frac{\psi\left(r a_{w}^{*}+w-v\right)+\tau Y(\tau)}{\sigma} .
\end{aligned}
$$

These equations can be written in the form

$$
\begin{aligned}
& \tau \frac{d X}{d \tau}+(1+A) X+B Y=c_{1}(v)+\tau f(\tau, X, Y, v), \\
& \tau \frac{d Y}{d \tau}+Y=c_{2}(v)+\tau g(\tau, X, Y, v),
\end{aligned}
$$

where $f$ and $g$ are smooth functions, $A=\frac{s \psi^{-\sigma}}{r \sigma}>0, B$ is a constant and $c_{1}$ and $c_{2}$ smooth functions of $v$ whose exact values are not important. This is a Fuchsian system ${ }^{22}$ and can therefore be treated using the following existence theorem which concerns a system of the form

$$
s \frac{d f}{d s}+N f=s G(s, f(s))+g(s) .
$$

Theorem A.24 (Rendall and Schmidt, 1991) Let $V$ be a finite-dimensional real vector space $N: V \rightarrow V$ a linear mapping, $G: V \times I \rightarrow V$ a smooth mapping and $g: I \rightarrow V$ a smooth mapping, where $I$ is an open interval in $\mathbf{R}$ containing zero. Consider the equation (A.32) for a function $f$ defined on a neighbourhood of 0 in $I$ and taking values in $V$. Suppose that each eigenvalue of $N$ has a positive real part. Then there exists an open interval $J$ with $0 \in J \subset I$ and a unique bounded $C^{1}$ function $f$ on $J \backslash\{0\}$ satisfying (A.32). Moreover $f$ extends to a $C^{\infty}$ solution of (A.32) on $J$. If $N, G$ and $g$ depend smoothly on a parameter $z$ and the eigenvalues of $N$ are distinct then the solution also depends smoothly on $z$.

Note that the solution whose existence is asserted by the theorem satisfies $N f(0)=g(0)$. Evidently the evolution equations for $X$ and $Y$ define a system of the form (A.32). It follows that this system has a unique smooth solution defined in a neighbourhood of $\tau=0$ and that this solution satisfies $(1+A) X(0)+b Y(0)=c_{1}$ and $Y(0)=c_{2}$. Thus the original system for $x$ and $y$ has a smooth solution in a neighbourhood of $a=a_{w}^{*}$ which satisfies the desired boundary conditions for $a=a_{w}^{*}$. In fact this is the unique solution satisfying these conditions since it can be checked that for any smooth solution $(x, y)$ with the desired boundary conditions the corresponding functions $(X, Y)$ are bounded close to $a=a_{w}^{*}$. The solution obtained in this way depends smoothly on the parameters $a_{w}^{*}$ and $v$.

It was proved in the last section that for $v>0$ the equations for $x$ and $y$ have a solution which is smooth on $\left(-b / a, a_{w}^{*}(v)\right)$ and satisfies the desired limiting conditions at the ends of the interval. Consider now a sequence $v_{n}$ of positive real numbers which tends to zero as $n \rightarrow \infty$. For each $n$ we obtain a solution $\left(x_{n}, y_{n}\right)$ satisfying the equation with parameter $v_{n}$ and the desired boundary conditions. Solving the Fuchsian system gives a two-parameter family $(x, y)$ of solutions with parameters $\left(v, a_{w}^{*}\right)$ arising from a two-parameter family $(X, Y)$ of solutions of

\footnotetext{
${ }^{22}$ For more information on Fuchsian systems in general the reader is referred to Kichenassamy, 2007.
} 
the Fuchsian system. The value $a_{w, n}^{*}$ of the parameter $a_{w}^{*}$ for the solution $\left(x_{n}, y_{n}\right)$ depends on $n$. The sequence $a_{w, n}^{*}$ is bounded since by construction it is contained in the interval $\left[-\frac{b}{r}, \frac{\psi[w-v]-b}{r[1-\psi]}\right]$ and a fortiori in the interval $\left[-\frac{b}{r}, \frac{\psi w-b}{r[1-\psi]}\right]$. It follows that by passing to a subsequence we can assume that $a_{w, n}^{*}$ tends to some $a_{w, \infty}^{*}$ for $n \rightarrow \infty$. Then $a_{w, n}^{*}+\frac{b}{r}$ converges to $a_{w, \infty}^{*}+\frac{b}{r}$. It follows that if $X_{n}$ and $Y_{n}$ are the solutions of the Fuchsian system corresponding to $x_{n}$ and $y_{n}$ then $\left(X_{n}, Y_{n}\right)$ converges uniformly to $(X, Y)$ on each interval of the form $[0, \eta]$ for $\eta$ positive and less than $a_{w, \infty}^{*}+\frac{b}{r}$. On the interval $\left[0, a_{w, \infty}^{*}+\frac{b}{r}\right)$ the functions $X$ and $Y$ are decreasing and bounded below. Hence they converge to some limiting values $X^{*}$ and $Y^{*}$ as $\tau \rightarrow a_{w, \infty}^{*}+\frac{b}{r}$. If $Y^{*}=0$ then the solution $(x, y)$ corresponding to $(X, Y)$ has all the desired properties. We will now show that assuming $Y^{*}>0$ leads to a contradiction. In that case the right hand side of the equation for $y$ remains regular in the limit $a \rightarrow-b / r$. Hence $\frac{d y}{d a}$ is are bounded in a neighbourhood of $x=-b / r$, say by a constant $C$. Consider now the region $K$ defined by the inequalities $x>-b / r$, $0<y<2 w$ and $y \geq 2(r a+b)$. On the region $K$ we have $\frac{y}{y-r a-b} \leq \frac{2(w-b)}{r a+b}$. Thus as long as a solution remains in $K$ the quantity $\frac{y}{r a+b-y}$ is bounded. This gives a bound for the right hand side of the evolution equation for $y$. Increase the size of $C$ if necessary so that it is greater than this bound. Since $y$ is an increasing function $y(a) \geq Y^{*}$ for all $a$. Let $\xi$ be a positive number. Since $y_{n}(-b / r+\xi)$ converges to $y(-b / r+\xi)$ for $n \rightarrow \infty$ it follows that $y_{n}(-b / r+\xi) \geq \frac{1}{2} Y^{*}$ for $n$ sufficiently large. Choose $\xi$ small enough that $C \xi \leq \frac{1}{4} Y^{*}$ and $\xi \leq Y^{*} / 4 r$. Then $y_{n}(a)$ remains in $K$ for all $a \in(-b / r,-b / r+\xi]$. It follows that $\bar{y}_{n}(-b / r) \geq \bar{y}_{n}(-b / r+\xi)-\frac{1}{4} Y^{*} \geq \frac{1}{4} Y^{*}>0$, a contradiction.

\section{A.6 Proof of Proposition 4.4}

(a) $c(a, w), c(a, b)$ is given as the solution of the reduced system (7), see also (A.13) for a more compact version. The right hand side in (A.13) is locally Lipschitz in the interior of the domain. Now fix some point $a_{0}$ in $]-b / r, a_{w}^{*}$ [ and consider the initial value problem (A.13) as started in $a_{0}$, i.e., for the domain $\left[a_{0}, a_{w}^{*}[\right.$. By the standard existence and uniqueness result for ODEs, the solution is $C^{1}$ in $a$ on this domain. On the other hand, $(c(a, w), c(a, b))$ also solves an ODE system on $\left.]-b / r, a_{0}\right]$ backward in the $a$ direction with right hand side simply obtained by changing the sign of the right hand side of (A.13). Hence, the solution is also $C^{1}$ on $\left.]-b / r, a_{0}\right]$.

(b) Let us concentrate on $z=w$. The reduced system (A.13) and the definition of $a_{w}^{*}$ shows that $c(a, w)<r a+w$ for $a<a_{w}^{*}$. Hence, the claimed monotonicity follows.

(c) For any $\left.a_{0} \in\right]-b / r, a_{w}^{*}$, the right hand side of the ODE for $\psi_{z}$ is locally Lipschitz (until the boundary is hit), hence we have continuity in the initial value by standard results. Furthermore, there is no jump when the boundary is hit, see $(\mathrm{d})$. For the boundary, we have to distinguish cases. If $z=b$ and $a_{0}=-b / r$, then $\psi_{z} \equiv-b / r$, so continuity holds. On the other hand, for $a_{0}=a_{w}^{*}$, we note that $c(a, b)$ - unlike $c(a, w)$ - is regular at $a_{w}^{*}$, as the right hand side of the second equation in (A.13) is uniformly Lipschitz around $a=a_{w}^{*}$, even if the derivative of $c(a, w)$ may explode. Hence, $a_{0} \mapsto \psi_{b}\left(a_{0}, t\right)$ is continuous on the whole domain. The proof for $z=w$ is analogous.

(d) The system (7) has a unique solution if two boundary conditions are added. One boundary condition requires $c(-b / r, b)=0$ from (4). The other boundary condition follows from (11), which is a property of the TSS in (9) and $\left(c\left(a_{w}^{*}, w\right) / c\left(a_{w}^{*}, b\right)\right)^{\sigma}=1-\frac{r-\rho}{s}$ from (8). The ODE system (7) with these three conditions fixes a unique path $c(a, z)$ plus $a_{w}^{*}$. The first property, $\lim _{t \rightarrow \infty} \psi_{w}\left(a_{0}, t\right)=a_{w}^{*}$, follows from standard properties of a saddle path: Assume the TSS lies to the left of $a_{w}^{*}$. Then (7) would imply that wealth still increases at this assumed TSS, leading to a contradiction. If the TSS lay to the right, wealth would fall, leading also to a contradiction. The second property follows from conceptionally identical arguments, only that 
the TSS cannot lie below $-b / r$ by assumption. ${ }^{23}$

\section{B Referees' appendix}

The Referees' appendix is available at www.waelde.com/pub.

\section{References}

Achdou, Y., F. Buera, J. Lasry, P. Lions, and B. Moll (2014): "Partial differential equation models in macroeconomics," Philosophical Transactions of the Royal Society A 372: 20130397, pp. $1-19$.

Achdou, Y., J. Han, J. Lasry, P. Lions, and B. Moll (2017): "Income and Wealth Distribution in Macroeconomics: A Continuous-Time Approach," mimeo Princeton University.

Ahn, S., G. Kaplan, B. Moll, T. Winberry, and C. Wolf (2018): "When Inequality Matters for Macro and Macro Matters for Inequality," NBER Macroeconomics Annual, forthcoming.

Aiyagari, S. R. (1994): "Uninsured Idiosyncratic Risk and Aggregate Saving," Quarterly Journal of Economics, 109, 659-84.

Anderson, R., and R. Raimondo (2008): "Equilibrium in Continous-Time Financial Markets: Endogenously Dynamically Complete Markets," Econometrica, 76(4), 841-907.

Aoki, S., and M. Nirei (2016): "Pareto distribution of income in neoclassical growth models," Review of Economic Dynamics, 20, 25-42.

(2017): "Zipf's Law, Pareto's Law, and the Evolution of Top Incomes in the United States," American Economic Journal: Macroeconomics 2017, 9(3), 36-71.

Applebaum, D. (2004): Lévy processes and stochastic calculus, vol. 93 of Cambridge Studies in Advanced Mathematics. Cambridge University Press, Cambridge.

Azema, J., M. Duflo, and D. Revuz (1969): "Mesure invariante des processus de Markov recurrents.," Sem. Probab. III, Univ. Strasbourg 1967/68, Lect. Notes Math. 88, 24-33 (1969).

Bandi, F. M., and T. H. Nguyen (2003): "On the functional estimation of jump-diffusion models," Journal of Econometrics, 116(1-2), 293-328.

Barczyk, D., and M. Kredler (2014): "Altruistically motivated transfers under uncertainty," Quantitative Economics, 5(3), 705-749.

Bayer, C., and K. Wälde (2010a): "Matching and Saving in Continuous Time: Proofs," CESifo Working Paper 3026-A. 3026.

(2010b): "Matching and Saving in Continuous Time: Theory," CESifo Working Paper

Benhabib, J., A. Bisin, and S. Zhu (2016): "The Distribution of Wealth in The Blanchard-Yaari Model," Macroeconomic Dynamics, 20(2), 466-481.

\footnotetext{
${ }^{23}$ Note that our condition includes the case that $-b / r$ is reached in finite time. This is therefore consistent with the finding of a Dirac mass in the stationary distribution (Achdou et al., 2017).
} 
Bewley, T. (1986): Stationary Monetary Equilibrium with a Continuum of Independently Fluctuating Consumers.pp. 79-102. Contributions to Mathematical Economics in Honor of Gerard Debreu, Werner Hildenbrand and Andreu Mas-Collel (eds), Amsterdam: North- Holland.

Bismut, J.-M. (1975): "Growth and Optimal Intertemporal Allocation of Risks," Journal of Economic Theory, 10(2), 239-257.

Brock, W., and M. Magill (1979): "Dynamics under Uncertainty," Econometrica, 47(4), 843868.

Cao, D., and W. Luo (2017): "Persistent Heterogeneous Returns and Top End Wealth Inequality," Review of Economic Dynamics, 26, 301-326.

Chang, F.-R., and A. Malliaris (1987): "Asymptotic Growth under Uncertainty: Existence and Uniqueness," Review of Economic Studies, 54(1), 169-174.

Down, D., S. P. Meyn, and R. L. Tweedie (1995): "Exponential and Uniform Ergodicity of Markov Processes," Annals of Probability, 23, 1671 - 1691.

Hansen, L. P., and J. A. Scheinkman (2009): "Long-Term Risk: An Operator Approach," Econometrica, 77(1), 177-234.

Heathcote, J., K. Storesletten, and G. Violante (2009): "Quantitative Macroeconomics with Heterogeneous Households," Annual Review of Economics, 1, 319-354.

Hopenhayn, H., and E. Prescott (1992): "Stochastic Monotonicity and Stationary Distributions for Dynamic Economies," Econometrica, 60(6), 1387-1406.

Huggett, M. (1993): "The risk-free rate in heterogeneous-agent incomplete-insurance economies," Journal of Economic Dynamics and Control, 17, 953-969.

Huggett, M., and S. Ospina (2001): "Aggregate precautionary savings: when is the third derivative irrelevant?," Journal of Monetary Economics, 48, 373-396.

Kamihigashi, T., and J. Stachurski (2012): "An order-theoretic mixing condition for monotone Markov chains," Statistics \& Probability Letters, 82(2), 262-267.

(2013): "Stochastic Stabilty in Monotone Economies," Theoretical Economics, forthcoming.

Kaplan, G., B. Moll, and G. L. Violante (2018): "Monetary Policy According to HANK," American Economic Review, 108, 697-743.

Karatzas, I., and S. E. Shreve (1988): Brownian motion and stochastic calculus, vol. 113 of Graduate Texts in Mathematics. Springer-Verlag, New York.

Keller, G., S. Rady, and M. Cripps (2005): "Strategic Experimentation with Exponential Bandits," Econometrica, 73(1), 39-68.

Khieu, H., and K. Wälde (2018a): "Capital Income Risk and the Dynamics of the Wealth Distribution," mimeo Johannes Gutenberg University Mainz.

(2018b): "Capital Income Risk and the Dynamics of the Wealth Distribution," IZA Discussion Paper No. 11840.

Kichenassamy, S. (2007): Fuchsian reduction. Birkhäuser, Boston. 
Krantz, G., and H. R. Parks (2002): A primer of real analytic functions. Birkhäuser, Boston.

Leland, H. E. (1968): "Saving and Uncertainty: The Precautionary Demand for Saving," The Quarterly Journal of Economics, 82(3), 465-473.

Lippi, F., S. Ragni, and N. Trachter (2015): "Optimal monetary policy with heterogeneous money holdings," Journal of Economic Theory, 159, 339-368.

Lise, J. (2013): "On-the-Job Search and Precautionary Savings," Review of Economic Studies, $80,1086-1113$.

Magill, M. (1977): "A Local Analysis of N-Sector Capital Accumulation under Uncertainty," Journal of Economic Theory, 15(1), 211-219.

Mattheij, R., and J. Molenaar (2002): Ordinary differential equations in theory and practice, vol. 43 of Classics in Applied Mathematics. Society for Industrial and Applied Mathematics (SIAM), Philadelphia, PA, Reprint of the 1996 original.

Merton, R. C. (1975): "An Asymptotic Theory of Growth under Uncertainty," The Review of Economic Studies, 42(3), 375-393.

Meyn, S. P., and R. L. Tweedie (1993a): Markov chains and stochastic stability, Communications and Control Engineering Series. Springer-Verlag London Ltd., London.

Meyn, S. P., and R. L. Tweedie (1993b): "Stability of Markovian processes. II. Continuous-time processes and sampled chains," Adv. in Appl. Probab., 25(3), 487-517.

(1993c): "Stability of Markovian processes. III. Foster-Lyapunov criteria for continuoustime processes," Adv. in Appl. Probab., 25(3), 518-548.

Nuño, G., and B. Moll (2018): "Social optima in economies with heterogeneous agents," Review of Economic Dynamics, 28, 150-180.

Picard, J. (1995/97): "Density in small time for Levy processes," ESAIM Probab. Statist., 1, 357-389 (electronic).

Raimondo, R. C. (2005): "Market clearing, utility functions, and securities prices," Economic Theory, 25(2), 265-285.

Rendall, A. D., and B. G. Schmidt (1991): "Existence and properties of spherically symmetric static fluid bodies with a given equation of state.," Classical and Quantum Gravity, 8(5), 985-1000.

Rishel, R. (1970): "Necessary and Sufficient Dynamic Programming Conditions for Continuous Time Stochastic Optimal Control," SIAM Journal on Control and Optimization, 8(4), 559571.

Scheinkman, J., and L. Weis (1986): "Borrowing Constraints and Aggregate Economic Activity," Econometrica, 54(1), 23-45.

Wang, C., N. Wang, and J. Yang (2013): "Optimal Consumption and Savings With Stochastic Income," NBER Working Paper, 19319, 1-43.

Wang, N. (2007): "An equilibrium model of wealth distribution," Journal of Monetary Economics, 54(7), 1882-1904.

Wälde, K. (1999): "Optimal Saving under Poisson Uncertainty,” Journal of Economic Theory, $87,194-217$. 